\title{
TU/e EmonOWEN

\section{Topology optimization as a powerful tool to design advanced PEMFCs flow fields}

\section{Citation for published version (APA):}

Behrou, R., Pizzolato, A., \& Forner-Cuenca, A. (2019). Topology optimization as a powerful tool to design advanced PEMFCs flow fields. International Journal of Heat and Mass Transfer, 135, 72-92.

https://doi.org/10.1016/j.ijheatmasstransfer.2019.01.050

\section{Document license: \\ TAVERNE}

DOI:

10.1016/j.ijheatmasstransfer.2019.01.050

Document status and date:

Published: 01/06/2019

\section{Document Version:}

Publisher's PDF, also known as Version of Record (includes final page, issue and volume numbers)

\section{Please check the document version of this publication:}

- A submitted manuscript is the version of the article upon submission and before peer-review. There can be important differences between the submitted version and the official published version of record. People interested in the research are advised to contact the author for the final version of the publication, or visit the $\mathrm{DOI}$ to the publisher's website.

- The final author version and the galley proof are versions of the publication after peer review.

- The final published version features the final layout of the paper including the volume, issue and page numbers.

Link to publication

\section{General rights}

Copyright and moral rights for the publications made accessible in the public portal are retained by the authors and/or other copyright owners and it is a condition of accessing publications that users recognise and abide by the legal requirements associated with these rights.

- Users may download and print one copy of any publication from the public portal for the purpose of private study or research.

- You may not further distribute the material or use it for any profit-making activity or commercial gain

- You may freely distribute the URL identifying the publication in the public portal.

If the publication is distributed under the terms of Article 25fa of the Dutch Copyright Act, indicated by the "Taverne" license above, please follow below link for the End User Agreement:

www.tue.nl/taverne

Take down policy

If you believe that this document breaches copyright please contact us at:

openaccess@tue.nl

providing details and we will investigate your claim. 


\title{
Topology optimization as a powerful tool to design advanced PEMFCs flow fields
}

\author{
Reza Behrou $^{\mathrm{a}, *}$, Alberto Pizzolato $^{\mathrm{b}}$, Antoni Forner-Cuenca ${ }^{\mathrm{c}}$ \\ a Department of Mechanical and Aerospace Engineering, University of California San Diego, La Jolla, CA 92093, USA \\ ${ }^{b}$ Department of Energy, Politecnico di Torino, Corso Duca degli Abruzzi 24, Turin, Italy \\ ' Department of Chemical Engineering, Massachusetts Institute of Technology, Cambridge, MA 02139, USA
}

\section{A R T I C L E I N F O}

\section{Article history:}

Received 26 September 2018

Received in revised form 1 January 2019

Accepted 10 January 2019

Available online 1 February 2019

\section{Keywords:}

Fuel cell

Topology optimization

Gas flow channel

Adjoint sensitivity

Homogenized current density

Maximized output power

\begin{abstract}
A B S T R A C T
This paper presents a thorough and affordable numerical framework to design the flow field of Proton Exchange Membrane Fuel Cells using topology optimization. No assumption is made about the layout of the channels, which freely evolves along the optimization process, resulting in non-trivial optimized geometries. The optimization problem is formulated to maximize both the power generation and homogeneity of current density distribution, in the spirit of reduced costs and increased durability. The evolution of the flow field geometry is computed with a gradient-based optimizer with gradients of the objective and constraints computed through the discrete adjoint method. At each optimization iteration, the incompressible Navier-Stokes, advection-diffusion, and Butler-Volmer equations are solved with a 2D finite element model that predicts the flow in the channels, transport of chemical species and electrochemical reactions. The 3D transport effects are accounted for in the 2D model through an original depth-averaging procedure. The results of the $2 \mathrm{D}$ prediction are verified against a full $3 \mathrm{D}$ model calibrated using numerical and experimental results. The developed design framework can be used to identify flow field layouts that outperform current industrial solutions, catch design trends and provide guidelines to technology practitioners. The topology-optimized designs yield significant power generation enhancements, an improved reactant distribution and a reduced pressure drop as compared to conventional flow fields. Increasing the inlet pressure leads to more and more intricate configurations with complex topologies and highly tortuous channels. However, the cell performance is found to be more sensitive to the topology of the flow distributor at low rather than at high inlet pressures. Considering a measure of the current density homogeneity in the optimization objective allows the identification of layouts in which the gas channels concentrate close to the outlets rather than close to the inlet. These design features slightly affect the amount of power generated, suggesting a viable route for future technological development.
\end{abstract}

(c) 2019 Elsevier Ltd. All rights reserved.

\section{Introduction}

Proton Exchange Membrane Fuel Cells (PEMFCs) are electrochemical devices used to directly convert the chemical energy of hydrogen into electricity [1]. They represent an efficient power generation solution for both stationary and moving power systems such as automotive, aerospace and marine vehicles [2-4] because of their high power density, low operating temperature, low weight and fast startups [2,5]. However, their limited durability and high cost are major obstacles to achieve a large scale commercialization [6].

\footnotetext{
* Corresponding author.

E-mail address: rbehrou@eng.ucsd.edu (R. Behrou).
}

A schematic representation of the cathodic portion of a typical PEMFC is shown in Fig. 1. A graphite Bi-Polar Plate (BPP) lies on a porous Gas Diffusion Layer (GDL), which provides a path for the reactants to the active sites. The BPP serves as an electronic interconnection between contiguous cells and feeds air to the cathode and hydrogen to the anode. This is achieved through channels separated by graphite ribs. At the Polymer Electrolyte Membrane (PEM) interface, a thin Catalyst Layer (CL) is responsible for the recombination of oxygen with protons through a complex variety of electrochemical reactions and mass transfer phenomena. The BPP is a crucial component playing four major roles [7]: (i) it distributes the reactants homogeneously over the reactive sites in such a way that both hot and cold spots are avoided; (ii) it minimizes the pressure drop from the inlet to the outlet to avoid the installation of external blowers, limit parasitic power, cross- 


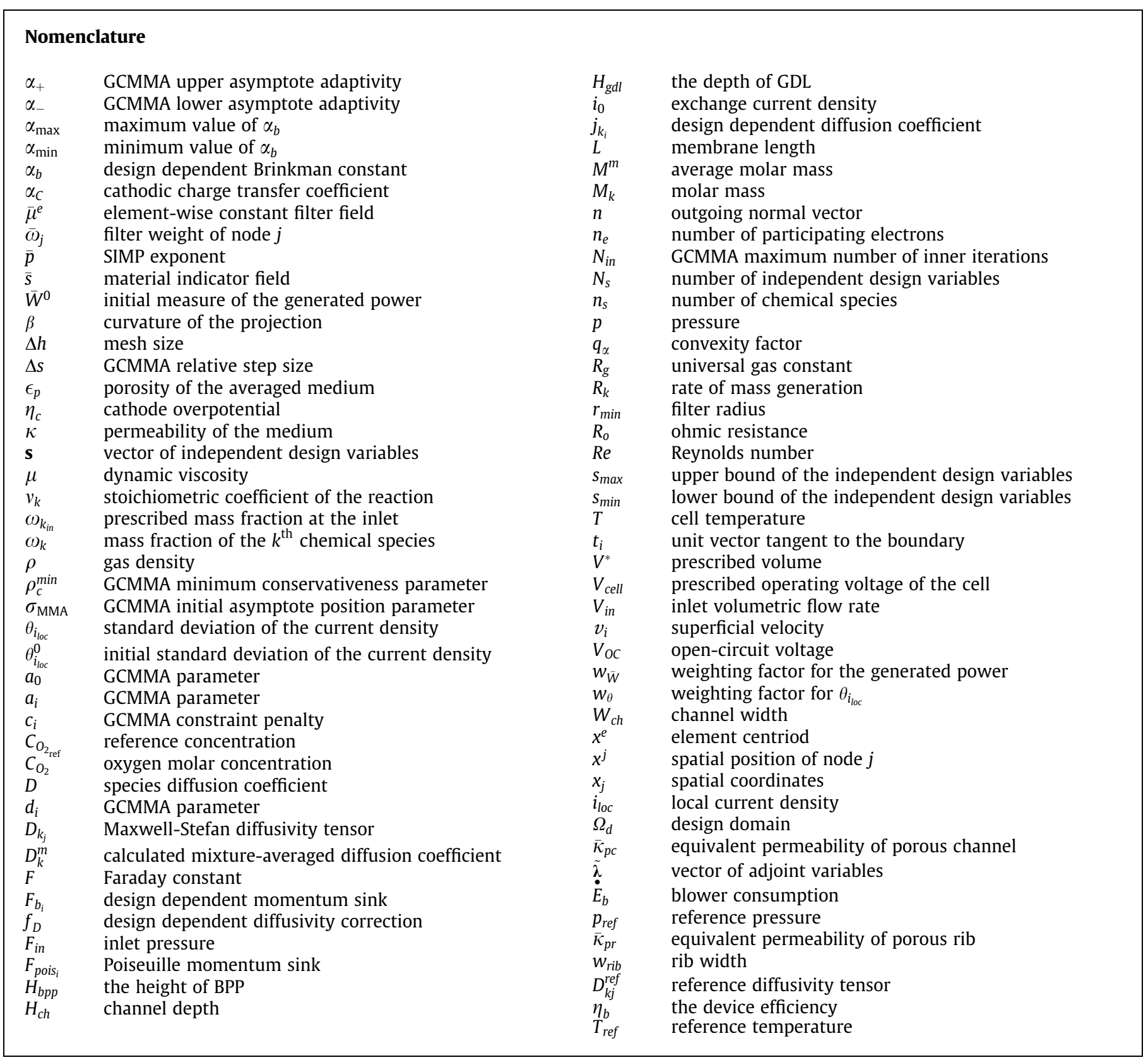

leakages of reactants and mechanical stresses, and control the homogeneous compression of the stack to avoid leaks, underutilized areas, and head management issues [8]; (iii) it facilitates water transport to avoid flooding; (iv) it provides a low electrical resistance. Hence, the geometry of the plate grooves affects the reactants transport, reactants utilization, reaction rate, and water management [4]. These factors largely influence the fuel cell performance [9]. For this reason, a vast body of literature investigated the effect of various geometrical features of the BPP. Research towards enhancement techniques can be grouped in two categories: design of the channels and ribs and design of the flow fields.

The design of the channels and ribs was tackled at different levels of complexity, ranging from the definition of the widths and depths to the shape optimization of the channel cross sections and utilization of tapered conduits. The definition of widths was generally considered using a channel-to-rib width ratio:

$\widetilde{W}=\frac{W_{c h}}{W_{\text {rib }}}$, where $W_{c h}$ and $W_{\text {rib }}$ represent the widths of the channels and ribs, respectively. Kumar and Reddy [5] were among the first to systematically investigate the effect of $\widetilde{W}$ on performance. Their results indicate that there might be an optimum value of $\widetilde{W}$ close to 3 . Many other studies were later conducted to assess the effect of this parameter on performance, see e.g. [10-12]. Most of the findings suggest that increasing $\widetilde{W}$ in general leads to a higher ohmic resistance and pressure drop, enhanced reactant distribution and improved water management. As observed by Manso et al. [13], only a limited number of studies focused on the effect of the channel depth, $H_{b p p}$. Afshari et al. [14] reported increased current densities for smaller depths and suggested to adopt $1 \mathrm{~mm}$ deep channels. A recent numerical and experimental study reported in [11] revealed that reduced channel depths and thinner ribs are always beneficial for water management. At low operating voltages, these features prevent the accumulation of water in the GDL through strong under-rib convection. At high operating voltages, water 

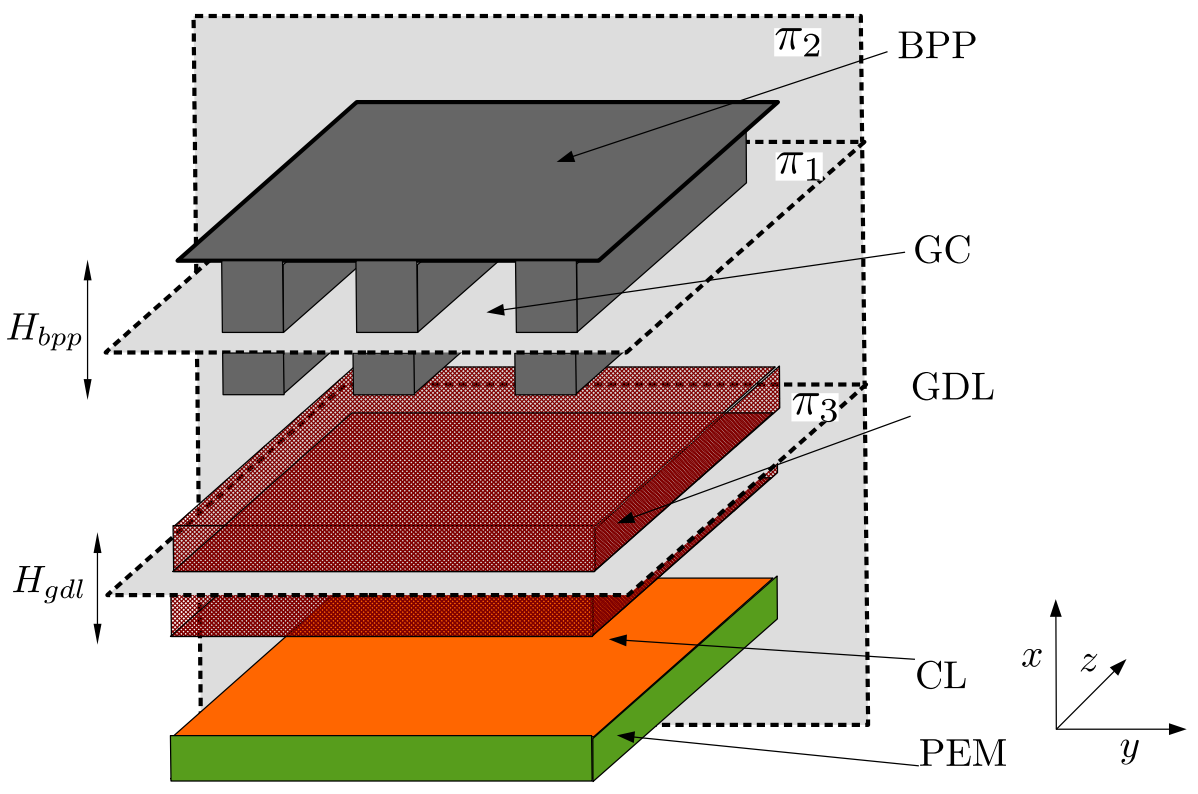

Fig. 1. Representative schematic of the cathodic portion of a PEMFC.

plugs in the channels are eliminated thanks to large streamwise pressure gradients.

All the studies discussed so far considered channels with rectangular cross-sections. Many studies dedicated to the search of alternative channel configurations such as hemispherical, triangular, trapezoidal and stepped cross sections. Ferng and Su [15] observed that designing a proper channel cross-section is crucial for parallel flow fields but yields minor effects in serpentine layouts. The authors of [5] suggested to use triangular and hemispherical channels to obtain the most effective mass distribution over the active sites but did not discuss any issue related to water management. Owejan et al. [16] and later Freire et al. [17] revealed that triangular and trapezoidal channels confine the accumulation of water at the channel angles in contact with GDL, yielding a little impact on the reactant distribution. Zeng et al. [18] used Genetic Algorithm (GA) to conduct a formal shape optimization of trapezoidal channels by varying the width of the bottom and top edges. Their optimized layout features a edge ratio of 1.45 and yields an increase of $8.09 \%$ in the output power compared to rectangular channels. The findings of the previous studies highlighted that water management and in turn reactant distribution are more critical in the regions of the cell located close to the outlet section. Building on this observation, many authors in the last decade focused on the design of tapered channels, i.e. with a varying cross-section along the path from the inlet to the outlet. The concept was first investigated numerically by Yan et al. [19] and Liu et al. [20]. The authors reported performance improvements for both width tapering, i.e. channel width increasing along the streamline direction, and height tapering, i.e. channel height decreasing along the streamline direction. Huang et al. [21,22] were the first to use formal shape optimization to optimize the height of the channel in a small portion close to the outlet section. The authors adopted second order B-splines with control points as design parameters. Later, Wang et al. [23] optimized the height of the channels of a serpentine configuration using 5 design variables. Their optimized geometry yields an $11.9 \%$ increase to the power generation as compared to a conventional non-tapered configuration. More recently, Mancusi et al. [24] observed a transition from a slug flow regime to a film flow regime at large tapering angles, which may lead to a performance degradation due to the reduced oxygen transport. This suggests that in general it is possible to identify an optimal tapering angle.

The search for optimal flow fields is an active area of research within the fuel cell community, as it is considered more promising than optimal channel design [25]. The most popular flow field configurations encompass parallel, serpentine, interdigitated, mesh, and bio-inspired flow fields [11], as shown schematically in Fig. 2. The serpentine and interdigitated layouts avoid water clogging and allow homogeneous reactant distribution. For these reasons, they have gathered most of the attention from technology practitioners [8]. However, their advantages come at the expense of a large pressure drop between the inlet and the outlet. On the other hand, the parallel flow fields guarantee a minimal pressure drop but are more susceptible to performance degradation due to

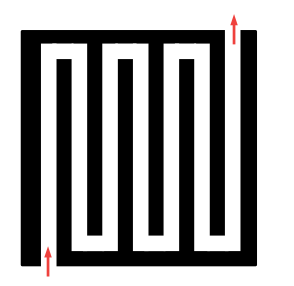

(a)

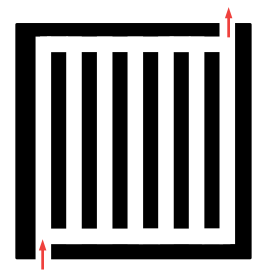

(b)

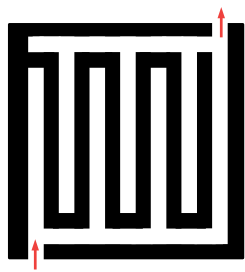

(c)

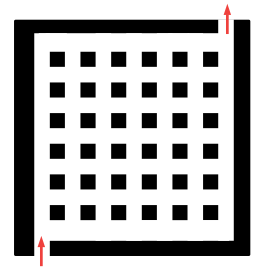

(d)

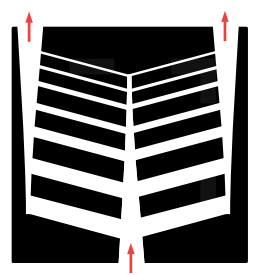

(e)
Fig. 2. Schematics of the most popular flow field geometries. The channels are indicated in white, the ribs are indicated in black; (a): serpentine; (b): parallel; (c): interdigitated; (d): mesh; (e): bio-inspired. 
flooding [26]. From these fundamental design concepts, research, and technology developed in a number of different directions. Many studies built on the serpentine concept. For instance, a concentric spiral layout used by Monsaf et al. [27] was found to yield an increased mass transfer at a reduced pressure drop. Alizadeh et al. [28] proposed a cascade layout where three serpentine channels with varying geometrical parameters are concatenated. Karthikeyan et al. [29] observed an improved water removal with the addition of porous carbon pins attached to the channels rib surface. Other studies, e.g. [30], reported an increased power density for wavy channels with sinusoidal width or convergent-divergent sections [31]. Fewer studies focused on the improvement of the parallel flow field concept. Imbrioscia and Fasoli [32] designed highly crafted baffles to improve the flow distribution. Lim et al. [8] reported an improved performance with multiple stages of distribution. A sub-channel connecting parallel flow paths for higher water removal was proposed by Wang et al. [11]. The mesh layout can be thought as a special case of the parallel flow field, in which the effect of clogging is reduced at no expenses of additional pressure drop [7]. A mesh-like flow pattern was obtained in Tsai et al. [33] and Huo et al. [34] using a metal foam as a flow distributor. For its Mirai model launched in 2014, Toyota refined the foam concept and developed a revolutionary 3D micro-lattice distributor [35] designed for maximized convective transport and water removal by capillary diffusion. Recently, some bio-inspired flow fields were proposed to mimic the flow distribution systems that are found in nature such as lungs [36] and leaves [37,38]. For a review of developments in this direction, the reader is referred to [7,39].

The presence of a multitude of largely different design concepts for BPPs suggests that current design procedures are inadequate for PEMFC systems. The small number of design parameters considered prevents dramatic design changes. Hence the initial choice of design parameters is crucial for the final geometry. The objective of this paper is to develop and demonstrate a method for the optimal design of flow fields of PEMFCs that requires no a priori assumptions on the layout. For this reason, we formulate the design process as a topology optimization problem. Topology optimization is a thorough and affordable form-finding methodology with applications covering a wide variety of engineering fields [40-43]. In the context of fuel cell systems design, Secanell et al. [25] were the first to spot the potential of topology optimization, claiming that it "can be used as an innovative method to obtain the optimal layout and geometry of the fuel cell flow field". To our best knowledge, only one previous study [44] has applied topology optimization to the design of PEMFC flow field. Although insightful, their study, like the heuristic based designs mentioned above, was limited in the physical phenomena which was included in the optimization. This valuable contribution relies on a simplified electrochemical model that uses a linear reaction rate in a single-component advection-diffusion equation. Design optimization was conducted only with respect to the output power with no explicit account of the current density homogeneity. The uniformity of the current density has important impacts on the performance of PEMFC. Unlike the non-uniform current density distribution that is responsible for thermal stresses of the membrane, leading to both performance and material degradation $[7,13]$ and can result in poor chemical reaction and catalyst utilization and reduced energy efficiency in the cell, the uniform current density distribution can be attributed to more uniform reactant gas and better water distributions [45]. Furthermore, the results presented by [44] suffers from limited resolution and convergence to well-defined manufacturable designs. Given these shortcomings, our work improves and expands upon the work of Kim and Sun [44]. The fluid-dynamic and electrochemical responses are computed with a 2D finite element framework that considers the incompressible Navier-Stokes, Butler-Volmer, and advectiondiffusion equations including mixture-averaged diffusion. The 3D transport effects are accounted for in the 2D model through an original depth-averaging procedure, which is verified against the predictions of a full 3D model calibrated using numerical and experimental results. The optimization problem is formulated considering both maximizing the output power and homogenizing the current density, in the spirit of reduced costs and increased durability. The design is evolved through a gradient-based optimizer using design sensitivities computed with the discrete adjoint method. The performance of the developed optimization framework is then evaluated by numerical experiments of interest for technological development.

The remainder of the paper is organized as follows. The physical model of the PEMFCs is described in Section 2. In Section 3, the formulation of the topology optimization problem is outlined. In Section 4, we first calibrate and verify our numerical model; then, we demonstrate the usefulness of the proposed topology optimization framework with selected numerical examples. Insights gained from the results and areas for future research are summarized in Section 5 .

\section{Physical model}

In this section, the theoretical and computational tools developed to compute the fluid-dynamic and electrochemical responses of the PEMFC systems are presented. First, the approach used to reduce the analysis of a three-dimensional problem in a 2D model is introduced. Then, the governing equations that rule the fluid flow, mass transport, and electrochemistry along with the suitable boundary conditions are discussed. Finally, we summarize the spatial discretization and numerical solution methods that we adopted. Although the impacts of liquid water represent an important step to accurately predict the numerical response and the optimized flow field design, this needs to develop a comprehensive numerical model, see for example [46], that describes capillary imbibition, interfacial phenomena, evaporation and condensation. For simplicity, we neglect the impact of liquid water in the current study.

\subsection{Depth-averaging}

We consider only the cathodic portion of the cell, which is the one showing the highest performance limitations [47]. We aim at finding an optimized layout of rectangular gas channels of height $H_{b p p}$ in the BPP. Due to its computational complexity, topology optimization greatly benefits from analyses in a reduced dimensionality. However, choosing a representative plane is not a trivial problem since the 3D transport effects are non-negligible. Considering a plane that crosses the GDL (such as $\pi_{3}$ in Fig. 1) does not allow the effect of the flow field geometry to be considered. Choosing plane $\pi_{1}$ (Fig. 1) would result in an inaccurate fluid response, as visible in Fig. 3(a). The impermeable graphite ribs filling $\Omega_{\text {rib }}$ would suppress the flow across contiguous channels. However, as shown in Fig. 3(c), mass transport across contiguous channels in 3D is possible thanks to the under-rib flow. For this reason, we use an averaging procedure that is inspired by [48]. Although contact resistance between the BPP and the GDL plays an important role on the performance of the PEMFC [49-51], for simplicity, the impacts of this phenomenon are neglected in the model. Fig. 3(c) shows a schematic representation of the process. We consider two Representative Volume Elements (RVEs) stretched along the $\mathrm{z}$ direction so that they span the entire depth of the GDL and BPP. One comprises the channel and GDL, the other comprises the rib and GDL. We aim at obtaining two equivalent two- 


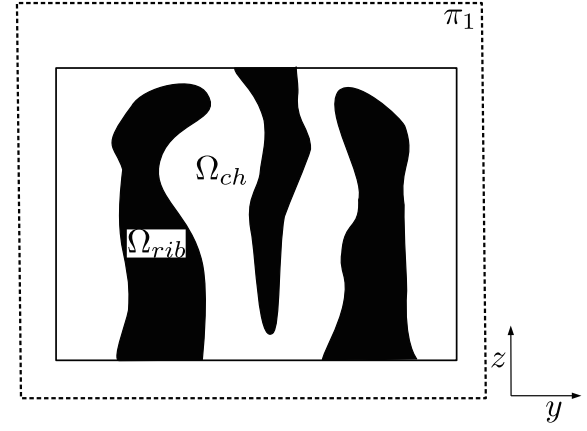

(a)
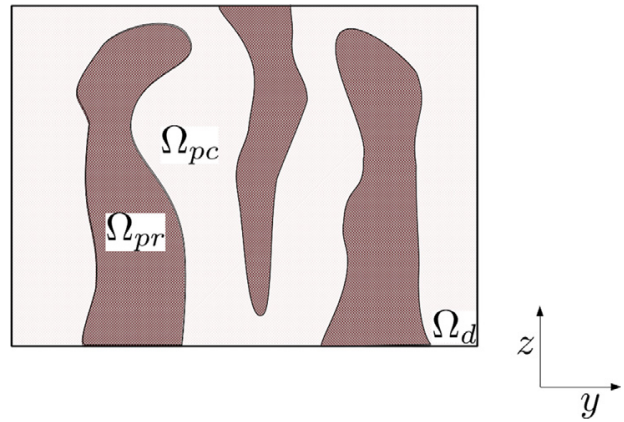

(b)

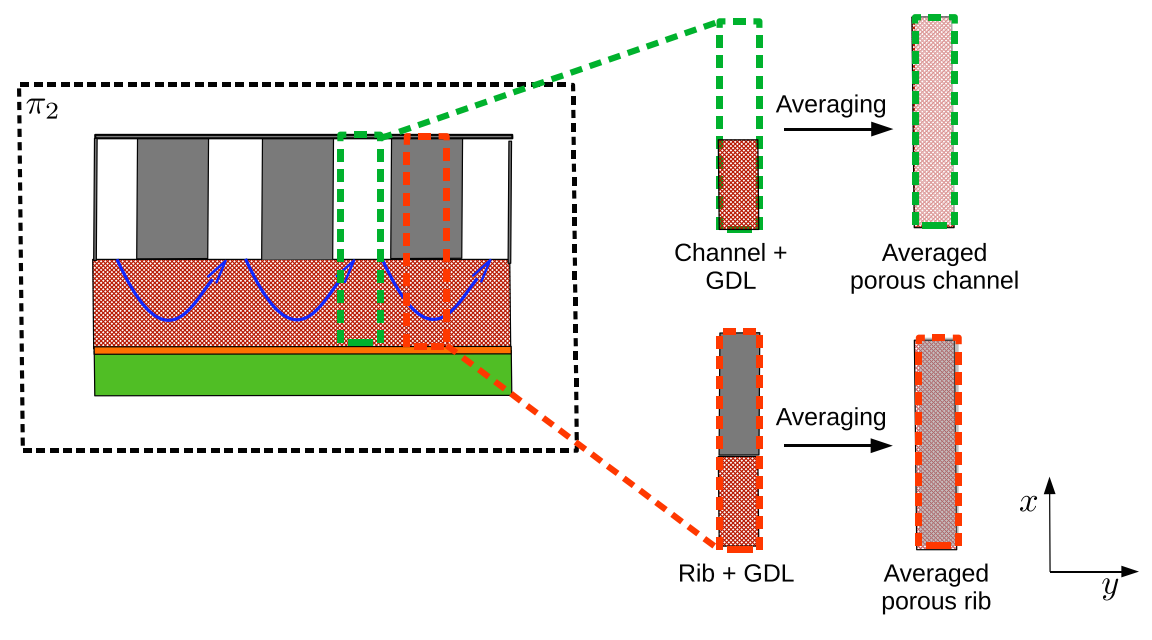

(c)

Fig. 3. (a): 2D plane leading to incorrect transport predictions; (b): final analysis and design domain; (c): averaging procedure.

dimensional porous media yielding the same transport response of the $3 \mathrm{D}$ system. This reduces to the calculation of the equivalent porosity, $\bar{\epsilon}_{p}$, permeability, $\bar{\kappa}$, and species diffusivity, $\bar{D}$ for both porous media. Similar to numerical homogenization approaches [52], a formal averaging requires that the size of the RVE is much smaller than the size of the macroscopic domain, $\Omega_{d}$. This condition does not hold here as the depth of our RVEs corresponds to the macroscopic dimension. For this reason, we follow a heuristic and simplified treatment according to the conventional engineering practice. Furthermore, the depth-averaged model accounts for convection and diffusion along zy but neglects out-of-plane transport. Hence, we rely on the assumption that this contribution can be approximated through bulk quantities. The accuracy of the framework will be verified in Sections 4.1 and 4.2. For stratified media with the main transport direction aligned with the strata, we can consider a simple depth-weighted mean of the transport properties [53] $\bar{\kappa}$ and $\bar{D}$. As the porosity is a bulk property, the same approach can be followed for the calculation of $\bar{\epsilon}_{p}$ [54]. Hence, for all the properties we write:

$\overline{(\cdot)}=\frac{(\cdot)_{g d l} H_{g d l}+(\cdot)_{b p p} H_{b p p}}{H_{b p p}+H_{g d l}}$,

where $(\bar{\cdot})$ indicates a generic averaged property for each of the equivalent porous media, $H$ denotes the depth along the $x$ direction and the subscripts "bpp" and "gdl" denote the BPP and the GDL, respectively. The property of the BPP, $(\cdot)_{b p p}$, can correspond to the one of the channels, $(\cdot)_{c h}$, or the one of the rib, $(\cdot)_{r i b}$. In the for- mer case we obtain an averaged property corresponding to a "porous channel", $(\bar{\cdot})_{p c}$; in the latter case, we obtain an averaged property corresponding to a "porous rib", $(\bar{\cdot})_{p r}$. This procedure leads to the computational and design domain depicted in Fig. 3(b). It is subdivided into two non-overlapping sub-domains, $\Omega_{p c}$ and $\Omega_{p r}$, such that $\Omega_{d}=\Omega_{p c} \cup \Omega_{p r}$, where $\Omega_{p c}$ and $\Omega_{p r}$ refer to the portions of the design domain occupied by the "porous channel" and the "porous rib", respectively.

\subsection{Governing equations}

Considering a steady-state and iso-thermal incompressible flow, the continuity equation in porous media can be written as:

$\frac{\partial v_{i}}{\partial x_{j}}=0$

where $v_{i}$ is the superficial velocity and $x_{j}$ is the spatial coordinates. The momentum transport along the $i^{\text {th }}$ direction is predicted by:

$$
\begin{aligned}
\frac{1}{\epsilon_{p}(\overline{\boldsymbol{s}})^{2}}\left(\rho v_{j} \frac{\partial v_{i}}{\partial x_{j}}\right)= & -\frac{\partial p}{\partial x_{i}}+\frac{1}{\epsilon_{p}(\bar{s})}\left(\frac{\partial}{\partial x_{j}}\left(\mu\left(\frac{\partial v_{i}}{\partial x_{j}}+\frac{\partial v_{j}}{\partial x_{i}}\right)\right)\right) \\
& +F_{\text {pois }_{i}}+F_{b_{i}}(\bar{s})=0
\end{aligned}
$$

where $p$ is the relative pressure, $\epsilon_{p}(\bar{s})$ is the design-dependent porosity of the averaged medium, $\rho$ is the gas density, $\mu$ is the dynamic viscosity, and $F_{\text {pois }_{i}}$ and $F_{b_{i}}(\bar{s})$ are a fixed and designdependent momentum sink, respectively. The design-dependent 
quantities are formulated as a function of $\bar{s}$, which is a material indicator field that defines the transition between the properties of the "porous rib" and those of the "porous channels", in such a way to account for geometry changes in the analysis. This field is controlled by the independent optimization variables, $s$, as described in Section 3. The Poiseuille momentum sink, $F_{\text {pois }}$, is needed in 2D analyses to account for the out-of-plane velocity gradients as follows [55]:

$F_{\text {pois }_{i}}=-12 \frac{\mu}{H_{\text {tot }}^{2}} v_{i}$

where $H_{t o t}=H_{g d l}+H_{b p p}$ is the depth of the electrode. The designdependent term, $F_{b_{i}}(\bar{s})$, is a Brinkman sink given by [56]:

$F_{b_{i}}(\bar{s})=-\alpha_{b}(\bar{s}) v_{i}$

where $\alpha_{b}(\bar{s})$ is the design-dependent Brinkman constant [56] that physically corresponds to $\alpha_{b}=\mu / \kappa$, with $\kappa$ denoting the permeability of the medium. The transport of the chemical species in the cell is modeled through an advection-diffusion-reaction equation:

$H_{t o t} \rho v_{i} \frac{\partial \omega_{k}}{\partial x_{i}}=H_{t o t} \frac{\partial j_{k_{i}}(\bar{s})}{\partial x_{i}}+R_{k}$,

where $\omega_{k}$ is the mass fraction of the $k^{\text {th }}$ chemical species with $k \in$ $\left[\mathrm{H}_{2} \mathrm{O}, \mathrm{N}_{2}, \mathrm{O}_{2}\right], j_{k_{j}}(\bar{s})$ is the design-dependent diffusive flux, and $R_{k}$ is the rate of mass generation due to electrochemical reactions. Diffusion is modeled through a mixture-averaged formulation:

$j_{k_{i}}(\bar{s})=\rho f_{D}(\bar{s}) D_{k}^{m} \frac{\partial \omega_{k}}{\partial x_{i}}+\rho \omega_{k} f_{D}(\bar{s}) D_{k}^{m} \frac{1}{M^{m}} \frac{\partial M^{m}}{\partial x_{i}}$,

where $f_{D}(\bar{s})$ is a design-dependent diffusivity correction, $M^{m}$ is the average molar mass computed as follows:

$M^{m}=\left(\sum_{k=1}^{n_{s}} \frac{\omega_{k}}{M_{k}}\right)^{-1}$

and $D_{k}^{m}$ is calculated using the mixture-averaged diffusion model given by the Wilke relation [57]:

$D_{k}^{m}=\frac{1-\omega_{k}}{\sum_{\substack{k=1 \\ k \neq j}}^{n_{s}} \frac{\omega_{k}}{D_{k j}}}$

where $n_{s}$ is the number of chemical species involved and $D_{k j}$ represents the Maxwell-Stefan diffusivity tensor calculated based on the reference values of binary diffusivities as follows [47]:

Table 1

Relevant physical parameters and properties of the PEMFC model.

\begin{tabular}{llll}
\hline Description & Symbol & Value & Unit \\
\hline Number of chemical species & $n_{s}$ & 3 & - \\
Number of participating electrons & $n_{e}$ & 4 & - \\
Molar mass of $\mathrm{H}_{2} \mathrm{O}$ & $M_{\mathrm{H}_{2} \mathrm{O}}$ & 18 & $\mathrm{~g} / \mathrm{mol}$ \\
Molar mass of $\mathrm{N}_{2}$ & $M_{\mathrm{N}_{2}}$ & 28 & $\mathrm{~g} / \mathrm{mol}$ \\
Molar mass of $\mathrm{O}_{2}$ & $M_{\mathrm{O}_{2}}$ & 32 & $\mathrm{~g} / \mathrm{mol}$ \\
Stoichiometric coefficient of $\mathrm{H}_{2} \mathrm{O}$ & $v_{\mathrm{H}_{2} \mathrm{O}}$ & 2 & - \\
Stoichiometric coefficient of $\mathrm{N}_{2}$ & $v_{\mathrm{N}_{2}}$ & 0 & - \\
Stoichiometric coefficient of $\mathrm{O}_{2}$ & $v_{\mathrm{O}_{2}}$ & -1 & - \\
Universal gas constant & $R_{g}$ & 8.3144 & $\mathrm{~J} / \mathrm{mol} \mathrm{K}$ \\
Faraday constant & $F$ & 96485.3 & $\mathrm{C} / \mathrm{mol}$ \\
Reference pressure & $p_{\mathrm{ref}}$ & 1 & $\mathrm{~atm}$ \\
Reference temperature & $T_{\mathrm{ref}}$ & 298.15 & $\mathrm{~K}$ \\
Oxygen diffusivity in water & $D_{\mathrm{O}_{2}, H_{2} \mathrm{O}}^{\mathrm{ref}}$ & $2.64 \times 10^{-5}$ & $\mathrm{~m}^{2} / \mathrm{s}$ \\
Nitrogen diffusivity in water & $D_{\mathrm{N}_{2}, H_{2} \mathrm{O}}^{\mathrm{ref}}$ & $2.64 \times 10^{-5}$ & $\mathrm{~m}^{2} / \mathrm{s}$ \\
Oxygen diffusivity in nitrogen & $D_{\mathrm{O}_{2}, N_{2}}^{\text {ref }}$ & $2.07 \times 10^{-5}$ & $\mathrm{~m}^{2} / \mathrm{s}$ \\
\hline
\end{tabular}

$D_{k j}=D_{k j}^{\mathrm{ref}}\left(\frac{p_{\text {ref }}}{p}\right)\left(\frac{T}{T_{\text {ref }}}\right)^{1.75} \quad$ with $\quad p=p_{\text {ref }}$,

where $D_{k j}^{\text {ref }}$ are the reference diffusivities, $p_{\text {ref }}$ is the reference pressure, and $T_{\text {ref }}$ is the reference temperature. The relevant physical parameters and properties of the PEMFC model are listed in Table 1. The mixture-averaged diffusion model yields computational savings as compared to the full Maxwell-Stefan formulation in that the diffusion fluxes are explicitly dependent on the species gradients. Although it was pointed out by [58] that Eq. (10) can be used only in the case of stagnant non-transferring mixtures, it is rather popular in the fuel cell community [59]. The results presented in [60] suggest that the choice of the diffusion model for the analysis of the PEMFC cathode yields negligible perturbations to the response predictions. Eq. (8) is solved for $n_{s}-1$ species. The mass fraction of the remaining chemical species is obtained using:

$\omega_{n_{s}}=1-\sum_{k=1}^{n_{s}-1} \omega_{k}$

The reaction term in Eq. (7) is written as:

$R_{k}=\frac{M_{k} v_{k} i_{l o c}}{n_{e} F}$

where $M_{k}$ is the molar mass, $v_{k}$ is the stoichiometric coefficient of the reaction, $n_{e}$ is the number of participating electrons, and $F$ is the Faraday constant. The local current density, $i_{l o c}$, is calculated through the Butler-Volmer equation [47]:

$i_{\text {loc }}=i_{0} \frac{C_{O_{2}}}{C_{O_{2_{\text {ref }}}}}\left(\exp \left(\frac{\alpha_{C}}{R_{g} T} F \eta_{c}\right)-\exp \left(-\frac{1-\alpha_{C}}{R_{g} T} F \eta_{c}\right)\right)$,

where $\alpha_{C}$ is the cathodic charge transfer coefficient, $T$ is the cell temperature, $R_{g}$ is the universal gas constant, $C_{\mathrm{O}_{2}}$ is the oxygen molar concentration and $C_{\mathrm{O}_{\text {ref }}}$ its reference value, $i_{0}$ is the exchange current density referred to the geometric area of the PEM, and $\eta_{c}$ is the cathode overpotential. Predicting the electrochemical response by (14) carries the assumption that the concentration of protons, electrons, and water does not limit the reaction rate [61]. The cathode overpotential is calculated as follows [62]:

$\eta_{c}=V_{O C}-V_{\text {cell }}-R_{o} i_{l o c}$,

where $V_{O C}$ is the open-circuit voltage, $V_{\text {cell }}$ is the prescribed operating voltage of the cell, and $R_{o}$ is the Ohmic resistance accounting for charge transport through the membrane and both the electrodes. The boundary conditions and characteristic dimensions of the considered domain are summarized in Fig. 4. We prescribe a fully developed flow at $\Gamma_{\text {out }}$ and a symmetry boundary condition along $\Gamma_{\text {sym }}$ such that:

$-\frac{\partial \omega_{k}}{\partial x_{i}} n_{i}=0 \quad$ on $\quad \Gamma_{\text {out }} \cup \Gamma_{\text {sym }}$,

where $n$ is the outward-pointing normal. The chemical composition of the mixture at the inlet section is known such that:

$\omega_{k}=\omega_{k_{\text {in }}} \quad$ on $\quad \Gamma_{\text {in }}$,

where $\omega_{k_{\text {in }}}$ is the prescribed mass fraction at the inlet. A no-slip condition is prescribed on the sealing gasket wall. Due to symmetry, only non-penetration is prescribed along the symmetry axis such that:

$v_{i}=\mathbf{0} \quad$ on $\quad \Gamma_{s g}$,

$v_{i} n_{i}=0 \quad$ on $\Gamma_{\text {sym }}$,

where $\Gamma_{\text {sg }}=\Gamma_{\text {tot }} /\left(\Gamma_{\text {sym }} \cup \Gamma_{\text {in }} \cup \Gamma_{\text {out }}\right)$ with $\Gamma_{\text {tot }}$ representing all the boundaries of the design domain. For our design optimization stud- 


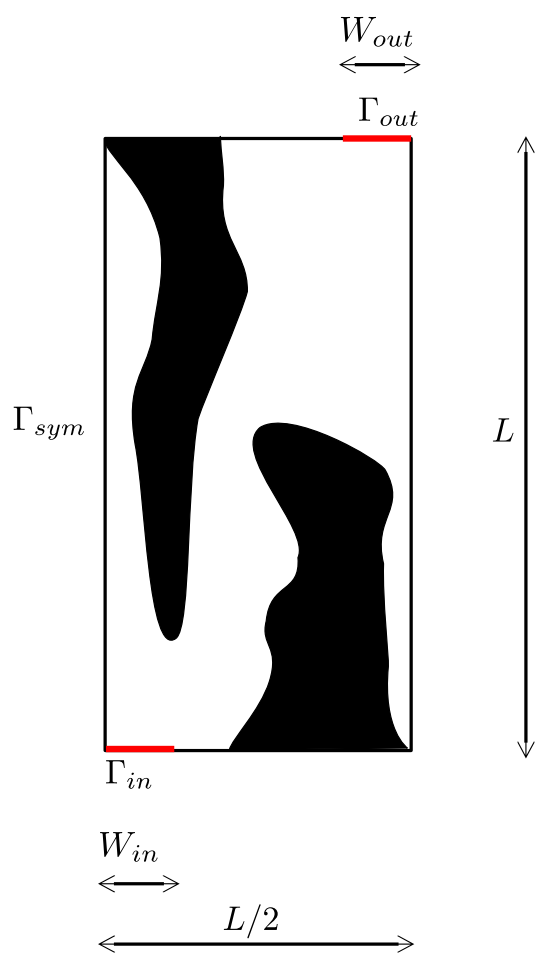

Fig. 4. Design domain and boundary conditions of the PEMFC model.

ies, a normal stress condition augmented with a nil tangential velocity condition is prescribed at the inlet as follows:

$n_{i} \sigma_{i j} n_{j}=F_{i n} \quad$ on $\quad \Gamma_{i n}$,

$v_{i} t_{i}=0 \quad$ on $\quad \Gamma_{i n}$,

where $F_{\text {in }}$ is the prescribed inlet pressure and $t_{i}$ is a unit vector tangent to the boundary. Note that Eq. (20) well approximates an inlet pressure condition for low-viscosity fluids. For our calibration and verification studies, we also consider an alternative inlet condition with fixed flow rate:

$v_{i} n_{i}=\frac{V_{i n}}{H_{t o t} W_{c h}} \quad$ on $\quad \Gamma_{i n}$,

where $V_{\text {in }}$ is the inlet volumetric flow rate. Condition (22) is augmented with the nil tangential velocity constraint (21). Vanishing normal and tangential stresses are prescribed at the outlet such that:

$\sigma_{i j} n_{j}=\mathbf{0} \quad$ on $\Gamma_{\text {out }}$.

\subsection{Numerical solution}

The numerical experiments presented in this paper are performed with COMSOL Multiphysics ${ }^{\circledR}$ [63]. The governing Eqs. (3), (4) and (7) are discretized in space using a mixed finite element model with four-node quadrilateral elements in 2D and tetrahedral elements in 3D. The velocity, pressure, and mass fractions are approximated by bilinear equal-order interpolations. Using equal-order approximation functions for the velocity and pressure does not satisfy the Babuska-Brezzi condition and leads to spurious oscillations in the pressure field [64]. Furthermore, high Peclet numbers and sharp crosswind gradients may result in overshoots and undershoots in the concentration field, leading to an unphysical response [65]. To prevent these numerical instabilities, the residuals of the weak form of the governing equations are aug- mented with consistent stabilization terms corresponding to Galerkin Least Squares (GLS) [66] and Cross-Wind Diffusion (CWD) [67]. For numerical analysis, the non-linear system of equations is solved with a damped Newton's method with the damping coefficient set to 0.7 . The discretized nonlinear problem is considered converged if the absolute residual norm drops below $10^{-7}$. The linear systems of equations arising at each Newton's iteration are solved with the MUltifrontal Massively Parallel sparse direct Solver (MUMPS) [68].

\section{Topology optimization}

In this section, we introduce the formulation of the topology optimization problem adopted for the design of the PEMFC flow fields. First, we present the topology optimization strategy used to describe the flow field geometry. Then, we define and discuss the objective and constraints. Last, we provide details related to the regularization scheme and numerical solution procedure used.

\subsection{Flow field geometry description}

In this paper, the layout of the flow field is controlled along the optimization process using a density-based topology optimization method [69]. The design-dependent properties depends on the material indicator field, $\bar{s}$, in such a way that:

$\left\{\alpha_{b}(\bar{s}), f_{D}(\bar{s}), \epsilon(\bar{s})\right\}=\left\{\begin{array}{ll}\left\{\alpha_{b_{p c}}, f_{D_{p c}}, \epsilon_{p c}\right\} & \text { if } \bar{s}=1 \\ \left\{\alpha_{b_{p r}}, f_{D_{p r}}, \epsilon_{p r}\right\} & \text { if } \bar{s}=0\end{array}\right.$.

Eq. (24) implies that $\bar{s}(\mathbf{x})=1$ denotes the "porous channel" portion of the design domain, i.e. $\mathbf{x} \in \Omega_{p c}$, and $\bar{s}(\mathbf{x})=0$ denotes the "porous rib" portion of the design domain, i.e. $\mathbf{x} \in \Omega_{p r}$ (Fig. 3(b)).

In density-based topology optimization, a continuous transition from the "porous rib" to the "porous channel" is allowed. This transforms a binary optimization problem, $\bar{s} \in\{0 ; 1\}$, into a continuous one, $\bar{s} \in[0,1]$, simplifying the computation of the gradients and rendering the optimization process more efficient [70]. The interpolation laws of the design-dependent properties should be formulated in such a way to make the intermediate nonphysical material, i.e. with $\bar{s} \in(0,1)$, inefficient such that a binary $\{0 ; 1\}$ layout is obtained in the optimized configuration. The Brinkman constant, $\alpha_{b}$, is interpolated as follows [71,72]:

$\alpha_{b}(\bar{s})=\alpha_{\min }+\left(\alpha_{\max }-\alpha_{\min }\right) \frac{1-\bar{s}}{1+q_{\alpha} \bar{s}}$,

where $q_{\alpha}$ is a convexity factor used to control the shape of the interpolation function at intermediate values of $\bar{s}, \alpha_{\max }$ and $\alpha_{\min }$ are the maximum and minimum values of $\alpha_{b}$, which serves for the purpose of recovering a porous media flow in the "porous rib" and "porous channel" portion of the design domain, respectively. These parameters are calculated as follows:

$\alpha_{\max }=\bar{\alpha}_{b_{p r}}=\frac{\mu}{\bar{\kappa}_{p r}}$

$\alpha_{\min }=\bar{\alpha}_{b_{p c}}=\frac{\mu}{\bar{\kappa}_{p c}}$

where $\bar{\kappa}_{p r}$ and $\bar{\kappa}_{p c}$ are the equivalent permeabilities of the "porous rib" and "porous channel" calculated using (2). Since $\bar{\kappa}_{p c}=\infty$, we would recover $\alpha_{\min }=\bar{\alpha}_{b p c}=0$ in the "porous channel". The designdependent diffusivity correction, $f_{D}$, is interpolated using the Solid Isotropic Material with Penalization (SIMP) method:

$f_{D}(\bar{s})=\bar{f}_{D_{p r}}+\left(\bar{f}_{D_{p c}}-\bar{f}_{D_{p r}}\right) \bar{s}^{\bar{p}}$ 
where $\bar{p}$ is the SIMP exponent, $\bar{f}_{D_{p r}}$ is the diffusivity correction factor of the "porous rib", and $\bar{f}_{D_{p c}}$ is the diffusivity correction factor of the "porous channel". These quantities are computed using the averaging law (2). The diffusivity factor in the GDL, $f_{D_{\text {gdl }}}$, is obtained from its porosity through the Bruggeman approximation [47]:

$f_{D_{g d l}}=\epsilon_{p_{g d l}^{3 / 2}}^{\text {. }}$

The porosity is interpolated linearly as follows:

$\epsilon_{p}(\bar{S})=\bar{\epsilon}_{p_{p r}}+\left(\bar{\epsilon}_{p_{p c}}-\bar{\epsilon}_{p_{p r}}\right) \bar{s}$,

where $\bar{\epsilon}_{p_{p r}}$ and $\bar{\epsilon}_{p_{p c}}$ are the equivalent porosities of the "porous rib" and the "porous channel", respectively, calculated using (2).

\subsection{Objectives and constraints}

We aim at distributing the reactant over the active sites in such a way that the most homogeneous current density field is created and the highest power generation is obtained at a limited pressure drop. We consider two optimization criteria: the standard deviation of the current density distribution, $\theta_{i_{l o c}}$, and the power output of the cell. The former is computed as follows:

$\theta_{i_{l o c}}=\sqrt{\frac{\int_{\Omega_{d}}\left(i_{l o c}-\bar{i}_{l o c}\right)^{2} d \mathbf{x}}{\int_{\Omega_{d}} d \mathbf{x}}}$,

with the average current density computed as follows:

$\bar{i}_{l o c}=\frac{\int_{\Omega_{d}} i_{l o c} d \mathbf{x}}{\int_{\Omega_{d}} d \mathbf{x}}$.

We formulate the multi-objective optimization problem using a penalty method as follows:

$$
\begin{aligned}
& \underset{\mathbf{s}}{\operatorname{minimize}} \frac{w_{\theta}}{\theta_{i_{l o c}}^{0}} \theta_{i_{l o c}}-\frac{w_{\bar{W}}}{\overline{W^{0}}} \int_{\Omega_{d}} V_{\text {cell }} \cdot i_{l o c} d \mathbf{x} \\
& \text { subject to } \quad \int_{\Omega_{d}} \bar{s} d \mathbf{x} \leqslant V^{*} \\
& \mathbf{s} \in \mathbf{S}=\left\{\mathbb{R}^{N_{s}} \mid s_{\min } \leqslant s_{i} \leqslant s_{\max }, i=1, \ldots, N_{s}\right\},
\end{aligned}
$$

where $\mathbf{s}$ is a vector collecting all the $N_{s}$ independent design variables, $w_{\theta}$ is the weighting factor for the standard deviation of the current density, $\theta_{i_{\text {loc }}}^{0}$ is the initial standard deviation of the current density, $w_{\bar{W}}$ is the weighting factor for the generated power, and $\bar{W}^{0}$ is the initial measure of the generated power. It should be noted that the maximization of the power requires a negative sign for the second term of the objective. The volume constraint prescribes that the total volume occupied by the "porous channels" (Fig. 3(c)) is less than a prescribed value, $V^{*}$. This avoids the trivial solution of having the entire design domain filled with "porous channel" material. Each design variable, $s_{i}$, is bounded with $s_{\min }$ and $s_{\max }$ set to magnitudes of zero and one, respectively.

\subsection{Sensitivity analysis}

The gradient-based optimization algorithm used in this paper relies on the gradients of the objective and constraints to drive the evolution of the flow field layout. In multi-physics topology optimization problems, the computational complexities of the sensitivities can be dramatically increased due to a large number of design-dependent physical variables. To provide a comprehensive sensitivity analysis with respect to the independent design variables, we adopt the discrete adjoint method. The sensitivity of a criterion, $\tilde{c}$, the objective or a constraint, with respect to a design variable, $s_{i}$, can be written as: $\frac{d \tilde{c}}{d s_{i}}=\frac{\partial \tilde{c}}{\partial s_{i}}+\frac{\partial \tilde{c}}{\partial \mathbf{u}} \frac{d \mathbf{u}}{d s_{i}}$

where $\mathbf{u}$ denotes the vector of unknown state variables that collects all the degrees of freedom of the discretized physical model. The total derivative of the state variables with respect to a design variable, $d \mathbf{u} / d s_{i}$, is computed from the total derivative of the converged residual equations as follows:

$\frac{d \mathbf{R}}{d s_{i}}=\frac{\partial \mathbf{R}}{\partial s_{i}}+\frac{\partial \mathbf{R}}{\partial \mathbf{u}} \frac{d \mathbf{u}}{d s_{i}}=\mathbf{0}$,

where $\mathbf{R}$ indicates the vector of residual equations that collect the weak form of the state equations given in (3), (4) and (7). From Eq. (35), substituting the expression for $d \mathbf{u} / d s_{i}$ into (34) yields:

$\frac{d \tilde{c}}{d s_{i}}=\frac{\partial \tilde{c}}{\partial s_{i}}+\tilde{\lambda}^{T} \frac{\partial \mathbf{R}}{\partial s_{i}}$

where $\tilde{\lambda}$ is a vector of adjoint variables computed by solving the following adjoint problem:

$\left(\frac{\partial \mathbf{R}}{\partial \mathbf{u}}\right)^{T} \tilde{\boldsymbol{\lambda}}=-\left(\frac{\partial \tilde{\boldsymbol{c}}}{\partial \mathbf{u}}\right)^{T}$.

\subsection{Regularization}

Using the independent design variable field, $s$, as the material indicator field to describe the geometry and interpolate the physical properties (Section 3.1) may result in numerical instabilities such as checkerboards and mesh-dependence of the optimized layouts. For this reason, several regularization approaches were proposed in the topology optimization community [73]. Among the possible options, filtering is no-doubt the most popular one as it solves both the above mentioned issues [73]. However, this operator blurs the boundary descriptions of the geometry, creating regions with the non-physical intermediate material, that does not disappear during the optimization process [74]. To reduce the inaccuracies introduced by filtering in the mechanical model, we adopt the Heaviside Projection Method (HPM) [75]. In the HPM, the filtered design variables are projected onto the finite element space by a regularized Heaviside function [75]. The filteringplus-projection regularization approach consists of two steps and relies on three different fields. The independent nodal design variables field, $s$, is first mapped onto an element-wise constant filtered field, $\bar{\mu}^{e}(s)$, using the following linear filtering scheme:

$\bar{\mu}^{e}(s)=\left(\sum_{j=1}^{\Omega_{\omega}} \bar{\omega}_{j}\right)^{-1} \sum_{j=1}^{\Omega_{\omega}} \bar{\omega}_{j} s_{j}$,

where $\Omega_{\omega}$ is the circular domain with radius $r_{\min }$ that is centered in the element centroid $x^{e}$ and $\bar{\omega}_{j}$ is the filter weight calculated as follows:

$\bar{\omega}_{j}=\left\{\begin{array}{ll}\frac{r_{\min }-\left\|x^{j}-x^{e}\right\|}{r_{\min }} & \text { if } x^{j} \in \Omega_{w} \\ 0 & \text { otherwise }\end{array}\right.$,

where $x^{j}$ is the position of node $j$. The elemental indicator field responsible for the geometry description, $\bar{s}^{e}$, is then related to the filtered design variables, $\bar{\mu}^{e}(s)$, as follows:

$\bar{s}^{e}\left(\bar{\mu}^{e}(s)\right)=1-e^{-\beta \bar{\mu}^{e}(s)}+\frac{\bar{\mu}^{e}(s)}{s_{\max }} e^{-\beta s_{\max }}$,

where $\beta \geqslant 0$ dictates the curvature of the projection that approaches the Heaviside function as $\beta \rightarrow \infty$ [75]. This regularization approach guarantees a minimum length scale of the "porous channels" [75]. 
Table 2

GCMMA parameters utilized in the optimization problems.

\begin{tabular}{lll}
\hline Description & Symbol & Value \\
\hline Relative step size & $\Delta s$ & 0.01 \\
Maximum inner iterations & $N_{\text {in }}$ & 2 \\
Initial asymptote position parameter & $\sigma_{\text {MMA }}$ & 0.5 \\
Lower asymptote adaptivity & $\alpha_{-}$ & 0.7 \\
Upper asymptote adaptivity & $\alpha_{+}$ & 1.43 \\
Constraint penalty & $c_{i}$ & 1000 \\
GCMMA parameter & $a_{i}$ & 0 \\
GCMMA parameter & $a_{0}$ & 1 \\
GCMMA parameter & $d_{i}$ & 0 \\
Minimum conservativeness parameter & $\rho_{c}^{\min }$ & $1 \times 10^{-5}$ \\
\hline
\end{tabular}

\subsection{Algorithmic summary of optimization problem}

The optimization problems presented in this paper are solved using COMSOL solvers linked with the Globally Convergent Method of Moving Asymptotes (GCMMA) optimizer [76] executed in MATLAB via LiveLink ${ }^{\mathrm{TM}}$ for MATLAB [63]. At each optimization iteration, the physical response of the system is computed using the numerical framework described in Section 2. The most relevant GCMMA parameters are summarized in Table 2. To discourage oscillatory behaviors for a large curvature of the regularization, $\beta$, the position of the initial asymptotes is modified using the formulation proposed by [77]. A continuation on the convexity factors, $q_{\alpha}$ and $\bar{p}$, is used to ensure a smooth evolution of the design and a stable convergence to satisfactory solutions. These parameters are modified during the optimization convergence as following:

$$
\begin{aligned}
& \bar{p}=\{1 ; 2 ; 3 ; 5\}, \\
& q_{\alpha}=\left\{10^{4} ; 10^{3} ; 10^{2} ; 10^{1}\right\} .
\end{aligned}
$$

The projection steepness parameter is kept constant at $\beta=50$. The transition to the next continuation step is performed every 60 iterations. The optimization procedure ends when all the continuation steps are completed and the convergence of the optimization process is verified after the final continuation step. The optimization problem is considered converged if the relative change of the objective function is less than $10^{-6}$ and the volume constraint is satisfied. We note that the continuation trajectories should be chosen carefully to avoid a strong imbalance between convective and diffusive transport at all continuation steps. For inlet pressures higher than $F_{\text {in }}>4 \mathrm{~Pa}$, we observed that strategy (41) leads to oscillations in the objective history; for $F_{\text {in }}>20$ Pa we also obtained unsatisfactory local minima. Skipping the first continuation step fixed the issue, yielding both a stable convergence and solutions with superior performance. For this reason, for all design studies with $F_{\text {in }}>4 \mathrm{~Pa}$ presented in Section 4, only the last three continuation steps are considered.

\section{Results and discussion}

In this section, we present and discuss the numerical results and design trends. Unless otherwise stated, for all the numerical results and optimized designs presented in the remainder of the paper, only half of the domain is considered as shown in Fig. 4. First, we calibrate a 3D physical model to a real system and verify the accuracy of our depth-averaging procedure by comparing $2 \mathrm{D}$ and 3D predictions. Then, we consider a reference design case with $F_{\text {in }}=5 \mathrm{~Pa}$ and compare its performance with conventional flow field layouts. Last, we investigate the effect of the inlet pressure and the weighting factor of the standard deviation of the current density on the performance of optimized designs of the PEMFC.

\subsection{Calibration and verification of the analysis model}

For calibration and verification purposes, we consider the PEMFC system investigated by [78]. It consists of a serpentine flow field with seven channels as shown in Fig. 5. The relevant parameters and properties are summarized in Tables 1 and 3.

The governing equations and boundary conditions presented in Section 2.2 are used to describe the fluid flow, mass transport, and electrochemistry in the 3D model. The system is fed by a mixture of oxygen and water using a fixed flow rate. Hence, we set $n_{s}=2$ and the velocity inlet boundary condition according to (22). Also, it is not possible to exploit symmetry in this numerical example, i.e. $\Gamma_{\text {sym }}=\varnothing$. We developed both a full 3D model and a reduced 2D model with properties computed through the averaging procedure described in Section 2.1. The 3D and 2D computational domains are meshed using free tetrahedral and free quadrilateral elements with characteristic size $\Delta h=2.7 \mathrm{~mm}$. This value is selected after a convergence study on the outlet oxygen flow rate obtained with $V_{\text {cell }}=0.4 \mathrm{~V}$ by comparing against the predictions computed on a reference mesh with $\Delta h=1.8 \mathrm{~mm}$. The results are summarized in Table 4. For the 3D numerical study, the characteristic mesh in the GDL is computed as $\Delta h_{g d l}=\max \left(\Delta h / 25, H_{g d l}\right)$. In [78], the electrochemistry at the anode was modeled using the Butler-Volmer equation and at cathode using a simplified Tafel equation [61]. To calibrate the model, the authors fitted the exchange current density to their experimental results. As our framework does not consider electrochemistry at the anode, we re-calibrate the exchange current density to their numerical results using a least-square fitting. For this process, we neglect the effect of the reactants concentration in (14). The computed value is $i_{0}=0.17 \mathrm{~A} / \mathrm{m}^{2}$. The polarization curves obtained using a full 3D and our reduced 2D analysis frameworks are compared to the numerical and experimental results given in [78] as shown in Fig. 6. This trend is obtained by sweeping the operating voltage of the cell, $V_{\text {cell }}$, in 10 steps from $0.2 \mathrm{~V}$ to $0.9 \mathrm{~V}$ and computing the average current density over the active area. Our 3D framework yields a mean relative deviation from the experimental and numerical results of $11.53 \%$ and $3.03 \%$, respectively. Most of the error is observed at $V_{\text {cell }} \geqslant 0.7 \mathrm{~V}$, which is far from the practical operating conditions of the cell targeting a peak power generation $\left(V_{\text {cell }} \sim 0.4 \mathrm{~V}\right)$. For $0.3 V \leqslant V_{\text {cell }} \leqslant 0.5 \mathrm{~V}$, we observe a satisfactory match between our 3D model and the numerical and experimental results of [78]. This indicates that our calibrated model is able to predict the electrochemical response of a real cell with sufficient accuracy in practical operating conditions. The polarization curve obtained using the 2D model closely approximates the one obtained in 3D. Although at low operating voltages we observe a slight overestimation of the generated current, we report a mean relative deviation of $0.65 \%$. This suggests that the oxygen distribution fields computed through the 2D and the 3D frameworks are comparable. Similarly to the results of [78], the polarization curve shows large activation losses at the low currents, caused by the slow reaction kinetics on the electrode surface. At higher current densities, ohmic polarization losses dominate. Since the cell resistance is constant, the polarization curve shows a linear trend. We also note the absence of mass transport losses at very high current densities. We believe the mass transport loss happens far away, at high current density. Due to the use of pure oxygen or ignoring the water transport phenomenon, our single-phase flow model cannot capture this phenomenon.

The most relevant state variable fields obtained using the 2D and 3D frameworks are qualitatively compared in Fig. 7 and Fig. 9, for $V_{\text {cell }}=0.4 \mathrm{~V}$. In 3D, we show the velocities and pressure distributions over two planes. In the portion of the domain occupied by the ribs, we consider plane $\pi_{3}$ (Fig. 1) located at 


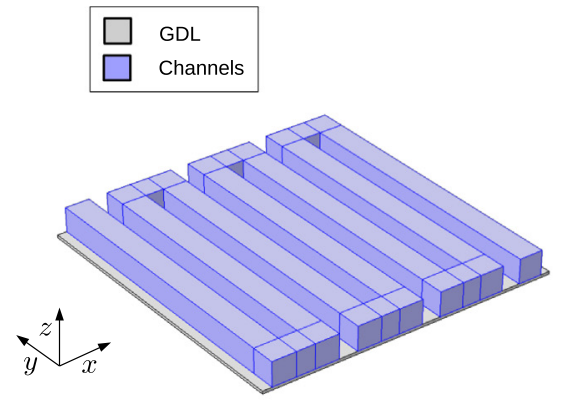

(a)

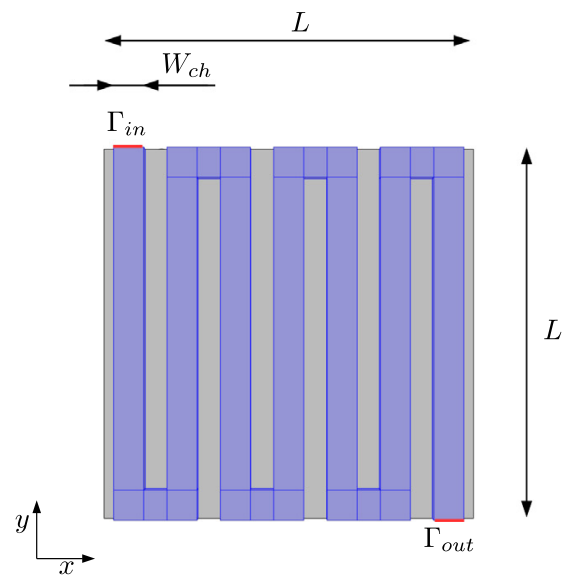

(b)
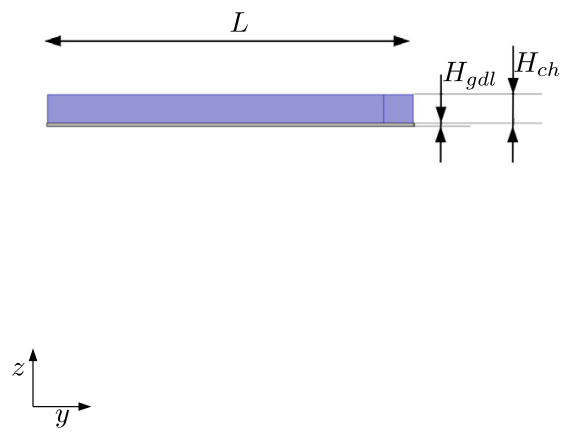

(c)

Fig. 5. Schematic representation of the 3D numerical study conducted for calibration and verification purposes. (a): 3D view; (b): top view; (c): side view.

Table 3

Material properties and model parameters adopted from [78].

\begin{tabular}{llll}
\hline Description & Symbol & Value & Unit \\
\hline Membrane length & $L$ & $2.236 \times 10^{-2}$ & $\mathrm{~m}$ \\
Channel width & $W_{c h}$ & $1.8 \times 10^{-3}$ & $\mathrm{~m}$ \\
Channel depth & $H_{c h}$ & $1.8 \times 10^{-3}$ & $\mathrm{~m}$ \\
GDL depth & $H_{g d l}$ & $0.2 \times 10^{-3}$ & $\mathrm{~m}$ \\
Open-circuit voltage & $V_{O C}$ & 1.22 & $\mathrm{~V}$ \\
Cell voltage & $V_{\text {cell }}$ & 0.40 & $\mathrm{~V}$ \\
Ohmic resistance & $R_{o}$ & $7 \times 10^{-5}$ & $\Omega \cdot \mathrm{m}^{2}$ \\
Cell temperature & $T$ & 343.15 & $\mathrm{~K}$ \\
Oxygen reference concentration & $C_{O_{2}}$ & 30 & $\mathrm{~mol} / \mathrm{m}^{3}$ \\
Inlet flow rate & $V_{\text {in }}$ & $2 \times 10^{-6}$ & $\mathrm{~m}{ }^{3} / \mathrm{s}$ \\
Cathodic transfer coefficient & $\alpha_{c}$ & 0.5 & - \\
Porosity of the GDL & $\epsilon_{G D L}$ & 0.8 & - \\
Inlet water mass fraction & $\omega_{H_{2} O_{\text {in }}}$ & 0.1 & - \\
Inlet oxygen mass fraction & $\omega_{O_{2 i n}}$ & 0.9 & - \\
Dynamic viscosity & $\mu$ & $2.07 \times 10^{-5}$ & $\mathrm{~Pa} \cdot \mathrm{s}$ \\
Gas density & $\rho$ & 1.0 & $\mathrm{~kg} / \mathrm{m}^{3}$ \\
Exchange current density & $i_{0}$ & 0.17 & $\mathrm{~A} / \mathrm{m}^{2}$ \\
Permeability of the GDL & $\kappa_{G D L}$ & $1.38 \times 10^{-11}$ & $\mathrm{~m}{ }^{2}$ \\
\hline
\end{tabular}

Table 4

Mesh convergence verification. The deviation is calculated with respect to a reference case with $\Delta h=1.8 \mathrm{~mm}$.

\begin{tabular}{lcc}
\hline Mesh size $\Delta h[\mathrm{~mm}]$ & 2D deviation [\%] & 3D deviation [\%] \\
\hline 7.2 & 5.68 & 4.50 \\
4.5 & 1.97 & 0.74 \\
2.7 & 0.64 & 0.27 \\
\hline
\end{tabular}

half-depth of the GDL. In the portion of the domain occupied by the channels, we consider plane $\pi_{1}$ located at half-depth of the BPP (Fig. 1). The velocity fields are compared in Fig. 7(a). The prediction of our 2D framework qualitatively matches those of the 3D model. The ratio between maximum velocities in $2 \mathrm{D}$ and $3 \mathrm{D}$ is $67.9 \%$, approximating the theoretical ratio of average-to-maximum velocity in plane Poiseuille flow of $66.6 \%$, in agreement with Eq. (5). Note that the 3D framework predicts velocity reductions after U-bends that are not captured in 2D. Similar trends in 2D and 3D are observed also for the pressure field shown in Fig. 7(b). The pressure diminishes gradually along the path from the inlet to the outlet. However, our 2D framework underestimates the pressure drop by $16.5 \%$. To assess how this difference can affect our fluiddynamic predictions, we compute the characteristic curve of the cell by sweeping the inlet flow rate, $V_{i n}$, from $10 \%$ to $150 \%$ of the design case. The results are shown in Fig. 8. The 3D model returns a super-linear pressure drop to flow rate relation. This trend is not reproducible in 2D under plane Poiseuille assumptions. We obtain a nearly linear relation using our 2D model. As the flow is laminar in the range of Reynolds number $8.7 \leqslant R e \leqslant 130.5$, we hypothesize that the super-linear drag is connected to geometry features that invalidate the fully-developed flow assumption, such as U-bends. Recall that we observed sudden velocity reductions in Fig. 7(a). To confirm this speculation, we compute the pressure drop over a single channel (the one connected to the inlet) and multiply the result by the number of channels to allow for comparisons. The 3D single-channel pressure drops closely match the 2D predictions. A high-fidelity 2D modeling of the 3D dynamics in serpentine flow fields would require the introduction of a quadratic Forchheimer drag term [79]. However, this term is highly 


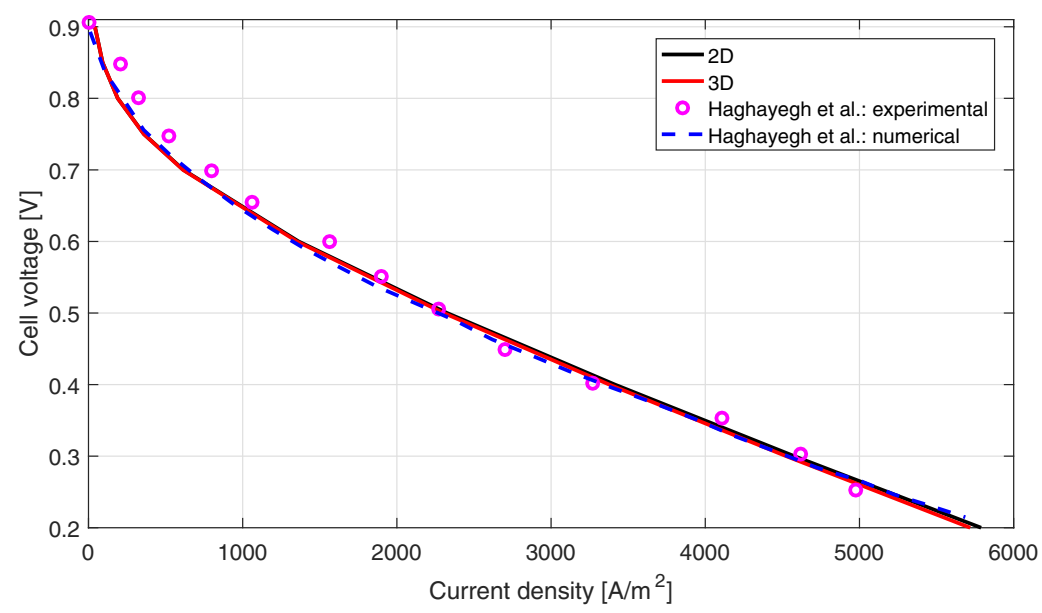

Fig. 6. Polarization curves obtained with the $2 \mathrm{D}$ and 3D models versus the experimental and numerical results of [78].

$2 \mathrm{D}$

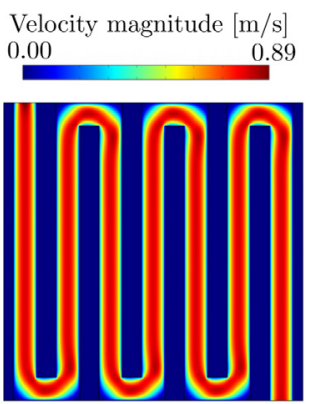

(a)

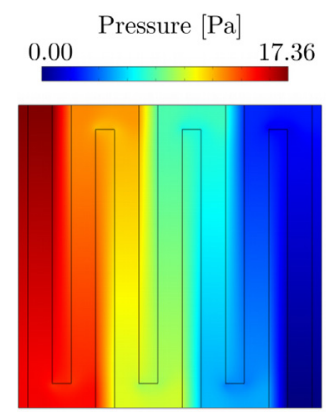

$3 \mathrm{D}$

Velocity magnitude $[\mathrm{m} / \mathrm{s}]$

$0.00 \quad 1.33$
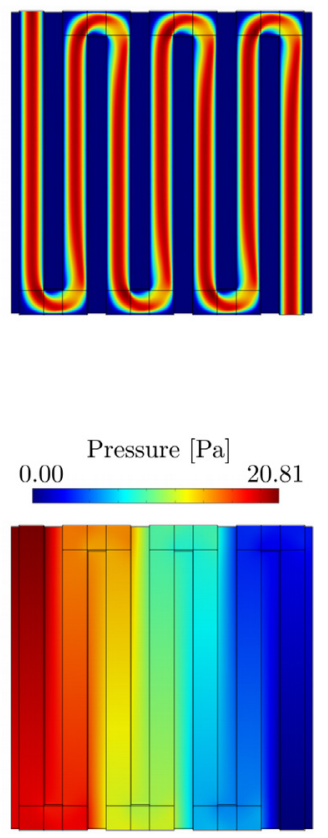

(b)

Fig. 7. Comparison of 2D and 3D responses. (a): velocity field; (b): pressure field.

geometry-dependent and hardly fits within an inverse design framework.

The oxygen mass fraction fields computed with the two frameworks for the serpentine geometry are compared in Fig. 9(a). In this case, 3D results are shown at the interface between GDL and catalyst, as these concentrations directly appear in the electrochemical model (14). We observe that the 2D and 3D predictions are qualitatively similar. The oxygen concentrates mainly along the channels. Local minima are visible in correspondence of the ribs since the limited convection and diffusion lead to a higher reactant depletion. The 3D model predicts a minimum oxygen fraction in a region close to the outlet. Due to our depth-averaging procedure, this value is overestimated in the $2 \mathrm{D}$ model, leading to a higher

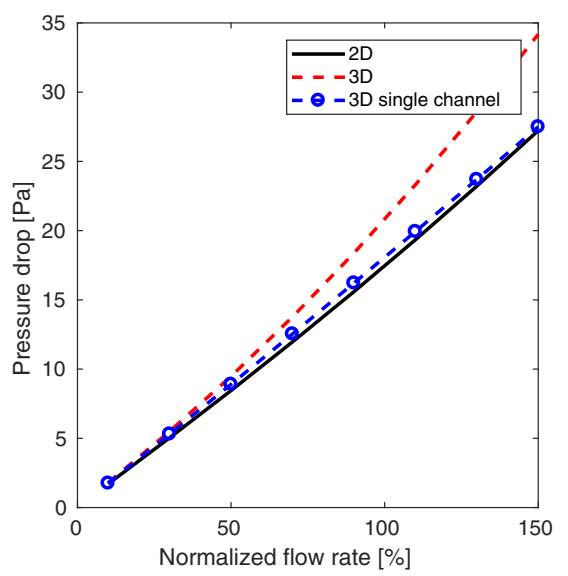

Fig. 8. Comparison of pressure drop using the 2D and 3D models.

current density generation. However, these results suggest that the 2D model is able to clearly represent the effect of the channel topology on the electrochemical kinetics of the cell. Hence, we consider the accuracy sufficient for this demonstrative study. To test whether this conclusion is affected by the channel layout, we modified the serpentine geometry to obtain a parallel flow field, which was then analyzed using both our 2D and 3D frameworks. The oxygen mass faction fields obtained are shown in Fig. 9(b) and confirm the trends identified for the serpentine layout. Note that for $\omega_{\mathrm{O}_{2}}=0.72$ we have $\omega_{\mathrm{H}_{2} \mathrm{O}}=0.28$, which is higher than the mass fraction that would be expected from a fully saturated oxygen stream at $343 \mathrm{~K}$, questioning the validity of a single-phase model. The comparison with experimental results presented in [78] suggests that the single-phase assumption yields accurate results for the system considered.

\subsection{Reference design}

In this section, we present the design optimization results obtained for a reference case with $F_{\text {in }}=5 \mathrm{~Pa}$ and $V_{\text {cell }}=0.4 \mathrm{~V}$ and compare its performance with conventional flow field layouts. The relevant model and optimization parameters of the reference design are given in Table 5 . The device is fed with air with $\omega_{\mathrm{O}_{2 \text { in }}}=0.228, \omega_{\mathrm{H}_{2} \mathrm{O}_{\text {in }}}=0.023$ and $\omega_{\mathrm{N}_{2 \text { in }}}=0.749$ [80]. Note that using an electrochemical model fitted for a mixture of oxygen and water (as obtained in Section 4.1) to predict the electrochem- 
$2 \mathrm{D}$

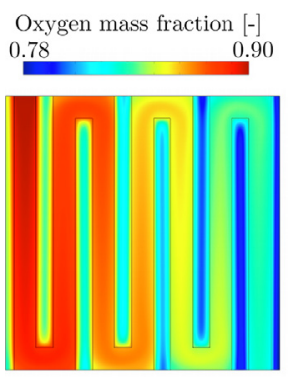

(a)

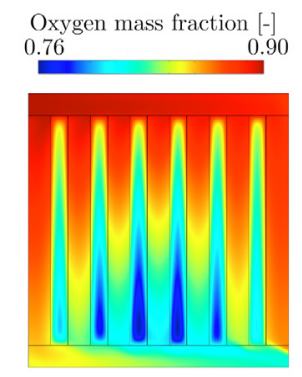

$3 \mathrm{D}$
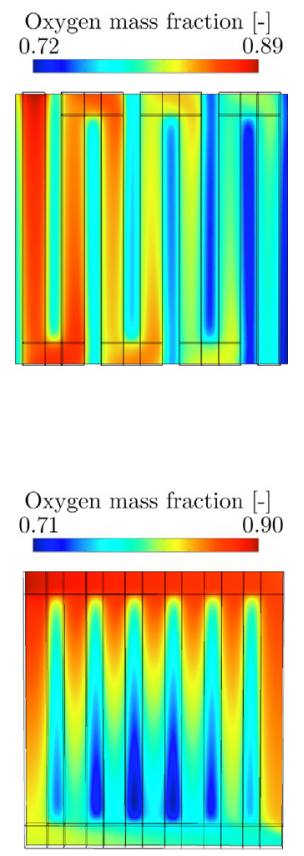

(b)

Fig. 9. Comparison of oxygen mass fraction fields. (a): Serpentine geometry; (b): parallel geometry.

Table 5

Relevant model and optimization parameters of the PEMFC reference design.

\begin{tabular}{llll}
\hline Description & Symbol & Value & Unit \\
\hline Membrane length & $L$ & $2.236 \times 10^{-2}$ & $\mathrm{~m}$ \\
Inlet width & $W_{\text {in }}$ & $1.2 \times 10^{-3}$ & $\mathrm{~m}$ \\
Outlet width & $W_{\text {out }}$ & $1.2 \times 10^{-3}$ & $\mathrm{~m}$ \\
Channel depth & $H_{c h}$ & $1.0 \times 10^{-3}$ & $\mathrm{~m}$ \\
GDL depth & $H_{g d l}$ & $0.38 \times 10^{-3}$ & $\mathrm{~m}$ \\
Prescribed inlet pressure & $F_{\text {in }}$ & 5 & $\mathrm{~Pa}$ \\
Prescribed cell voltage & $V_{c e l l}$ & 0.4 & $\mathrm{~V}$ \\
Inlet oxygen mass fraction & $\omega_{O_{2 i n}}$ & 0.228 & - \\
Inlet water mass fraction & $\omega_{H_{2} O_{i n}}$ & 0.023 & - \\
Inlet nitrogen mass fraction & $\omega_{N_{2 i n}}$ & 0.749 & - \\
Prescribed volume constraint & $V^{*}$ & $50 \%$ & - \\
Filter radius & $r_{m i n}$ & $0.35 \times 10^{-3}$ & $\mathrm{~m}$ \\
Equivalent permeability of the porous rib & $\bar{\kappa}_{p r}$ & $3.8 \times 10^{-12}$ & $\mathrm{~m}{ }^{2}$ \\
Equivalent permeability of the porous & $\bar{\kappa}_{p c}$ & $\infty$ & $\mathrm{m}{ }^{2}$ \\
$\quad$ channel & & & \\
Equivalent porosity of the porous rib & $\bar{\epsilon}_{p r}$ & 0.22029 & - \\
Equivalent porosity of the porous channel & $\bar{\epsilon}_{p c}$ & 0.94493 & - \\
Equivalent diffusivity correction factor the & $\bar{f}_{D_{p r}}$ & 0.19703 & - \\
$\quad$ porous rib & & & \\
Equivalent diffusivity correction factor the & $\bar{f}_{D_{p c}}$ & 0.92167 & - \\
$\quad$ porous channel & & & \\
\hline
\end{tabular}

ical response of a cell operated with air may be questioned in general. For the sake of this work, we consider this assumption valid. In this section, we aim at maximizing only the generated power. Hence, in Eq. (33) we set $w_{\theta}=0.00$ and $w_{\bar{w}}=1.00$. The design and computational domain corresponds to the one shown in Fig. 4 with $W_{\text {in }}=W_{\text {out }}=1.20 \mathrm{~mm}$ and $L=22.36 \mathrm{~mm}$ as in the previous section. The maximum volume of "porous channel" material, $V^{*}$, is set to $50 \%$ of the total. The other model and material properties are adopted from the model based parameters given in Table 1 and the verification study presented in Section 4.1. However, the channel and GDL depths are modified to produce all the topology optimization results. Since our analysis framework does not account for either flooding or dehydration, there is a chance that the optimized designs will not perform as expected. There is evidence that adopting small channel depths simplifies water management regardless of the flow field layout [11,14]. For this reason, we choose the configuration adopted in [80], where $H_{g d l}=0.38 \mathrm{~mm}$ and $H_{c h}=1.00 \mathrm{~mm}$. Note that water management is seldom an issue at higher temperatures [81]. Hence, the design results and trends presented hereafter apply to a hightemperature PEMFCs with little risk of bias due to physics reduction.

The objective history along with snapshots of the projected design variable field at selected design iterations are shown in Fig. 10. For all the designs presented in the remainder of the paper, we will adopt the colorbar presented in Fig. 10. The jumps in the objective history correspond to the transitions between the continuation steps. The initial design (Fig. 10(a)) corresponds to a homogeneous material indicator field with $\bar{s}=0.5$. Within the first continuation step, the "porous rib" material is retrieved from the regions close to the inlet and outlets and aggregated in two central areas. Four wide "porous channels" start to appear at iteration 20 (Fig. 10(b)), which then evolve into Y-shaped channel connections at iteration 60 (Fig. 10(c)). Some regions with intermediate $\bar{s}$ values are still present at the end of the first continuation step due to the moderate penalization of both the diffusive and advective transport. At iteration 100, as shown in Fig. 10(d), the design converges to a binary $\{0 ; 1\}$ layout since a stronger penalization is applied on the intermediate $\bar{s}$ values. From this point onward, limited objective and layout modifications are observed. The final optimized design after 180 iterations is shown in Fig. 11(a). The volume constraint is active in the optimized design, meaning that $50 \%$ of the design domain, $\Omega_{d}$, is occupied by the "porous channel" material. The optimized geometry shows features pertaining to different design concepts presented in Section 1. As in the parallel configuration, our optimized layout presents the inlet and outlet headers that are connected by a number of alternative paths. The intricate groove of flow channels somehow recalls the layout of the mesh flow fields. Furthermore, the width tapering of the main vertical channels and the inclination angle of the diagonal connections make it similar to the bio-inspired geometry proposed by [82] and schematically shown in Fig. 2(e). For this design optimization case, the need for the last continuation step could be questioned. However, this last step is essential to ensure convergence to binary $\{0 ; 1\}$ designs at higher $F_{\text {in }}$ values, as considered in the next section.

Fig. 11 shows a comparison of our optimized design with conventional flow channel layouts. Benchmark parallel and serpentine flow channel layouts are displayed in Fig. 11(d) and (g). For these geometries, the width of the channels, $W_{c h}$, is calculated so that the volume constraint in (33) is satisfied. The width of the inlet and outlets sections should be equal in all the layouts to allow for meaningful comparisons. Since $W_{c h}<W_{\text {in }}$ and $W_{c h}<W_{\text {out }}$, in the serpentine layout we created small convergent and divergent sections of length $W_{c h}$ at the inlet and outlets, respectively. The average current density, $\bar{i}_{\text {loc }}$, obtained with the three designs is summarized in Table 6 . The topology-optimized design yields superior performance resulting in a $5.7 \%$ and a $248.6 \%$ increase in the average current density as compared to the parallel and serpentine design, respectively. The velocity magnitude and the oxygen mass fraction fields displayed in Fig. 11 help to identify the physical reasons behind these performance differences. Due to the adopted kinetic model (Eq. (14)), the current density distribution fields closely resemble the oxygen mass fraction fields. The inspection of the colorbar range of the velocity field reveals that 


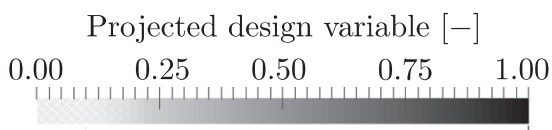

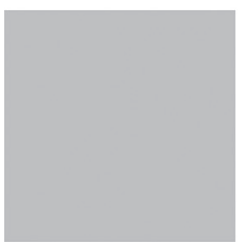

(a)

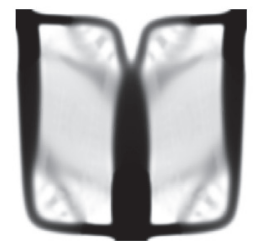

(b)

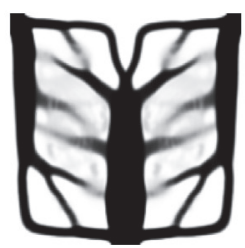

(c)

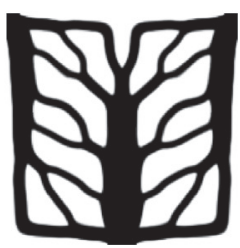

(d)

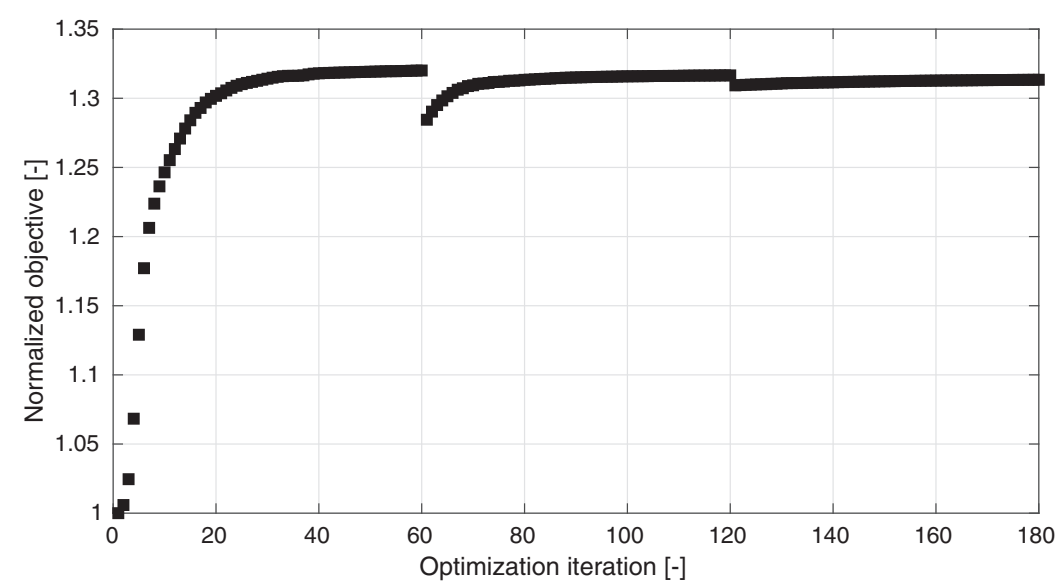

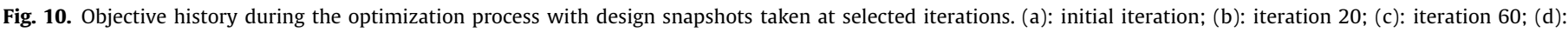
iteration 100 .

the average gas velocity in the serpentine layout is much smaller as compared to the other two alternatives. This is due to a higher average fluid-dynamic resistance of the gas paths linking the inlet to the outlets. Hence, the small amount of oxygen entering the cell is promptly depleted in a region close to the inlet resulting in a negligible amount of power generation in the remainder of the cell. A premature reactant depletion in single serpentine flow fields was also observed in [83]. In agreement with the considerations of [84], Fig. 11(e) suggests that the reactants are distributed ineffectively when using a parallel flow field. The uneven fluid-dynamic resistance among the parallel paths yields regions with large oxygen depletion so that the minimum oxygen mass fraction is $11.07 \%$. The flow distribution is more efficient when using our topologyoptimized design. The oxygen propagates in the cell following tree-like paths. This distribution strategy helps to avoid local minima of the reactant mass fraction field. The minimum oxygen mass fraction computed for the topology-optimized layout is $15.34 \%$. The single-phase assumption hardly affects the trends observed. The water molar fraction of a fully saturated stream is exceeded $(+13 \%)$ only by the benchmark serpentine design. For the reference topology-optimized and the benchmark parallel designs, we obtain a maximum water molar fraction $48 \%$ and $30 \%$ lower than the saturation value.

To make sure that the identified 2D design trends are not affected by our depth-averaging procedure, we analyze the alternative geometries in 3D. The 3D version of the topology-optimized layout is obtained using channel walls described by the $\bar{s}=0.5$ iso-countour. Views of the computed geometry and the 3D versions of the parallel and serpentine layouts are shown in Fig. 12. The three geometries are analyzed using high-resolution body-fitted meshes with 444113, 431012, 468174 tetrahedral elements, respectively. The average current densities computed in 3D are summarized in Table 7 . The results show a satisfactory match between the 2D and 3D predictions for the topology-optimized and parallel design. A larger discrepancy is computed for the serpentine layout due to the presence of quadratic drag effects in correspondence of $U$ bends that are not captured in 2D (see Section 4.1) and large oxygen depletion towards the outlet, yielding small catalyst-to-bulk mass fraction ratios. Despite this limited deviation, the 3D results confirm the design trends identified by the 2D model, suggesting that the depth-averaging procedure is a reliable approach for topology optimization purposes.

To complete the analysis, it is useful to identify the pressure drop required by the serpentine and parallel flow fields to yield a current density level comparable to the topology-optimized design. This allows the blower consumption, $\dot{E}_{b}$, of the three design alternatives to be cross-compared. This performance indicator is computed as follows:

$\dot{E}_{b}=\frac{\Delta p}{\eta_{b}} V_{\text {in }}$

where $\eta_{b}$ is the device efficiency, here set to 0.8 . Results are summarized in Table 8. Although blower consumption values are negligible for all the alternative configurations due to the small size of the system, the parallel and serpentine flow fields require roughly 3 times and 8 times more blower power than the topology-optimized design. Furthermore, as noted by Wang [85], these differences are 


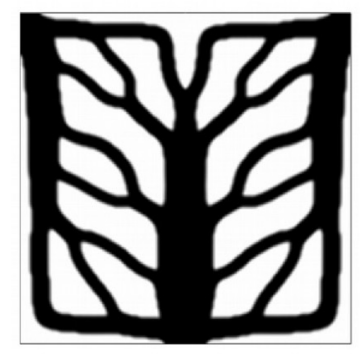

(a)

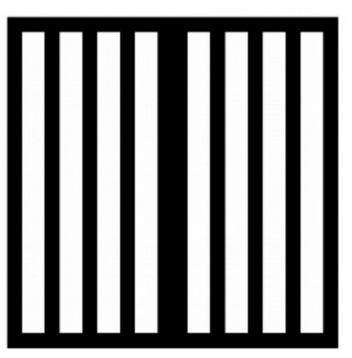

(d)

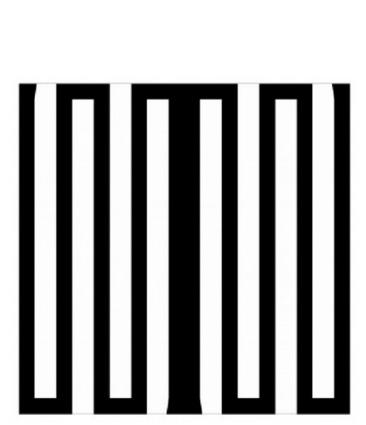

$(\mathrm{g})$
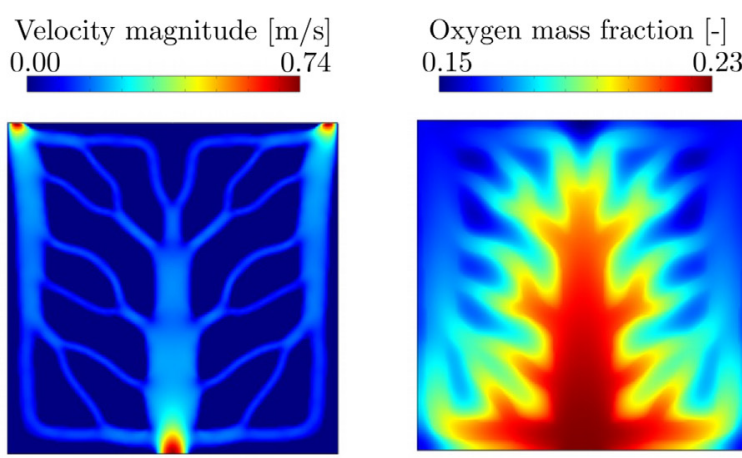

(b)

Velocity magnitude $[\mathrm{m} / \mathrm{s}]$ 0.00 0.58

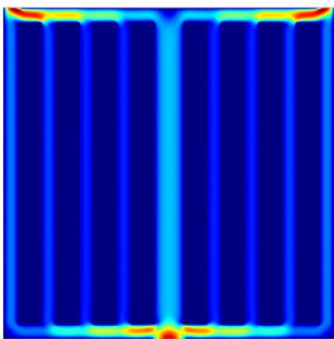

(e)

Velocity magnitude $[\mathrm{m} / \mathrm{s}]$ 0.00

0.10

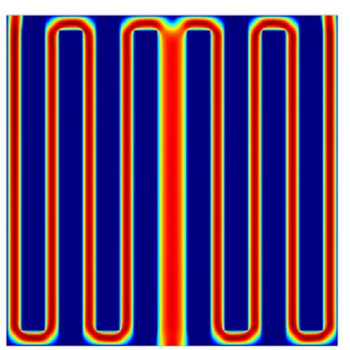

(h)

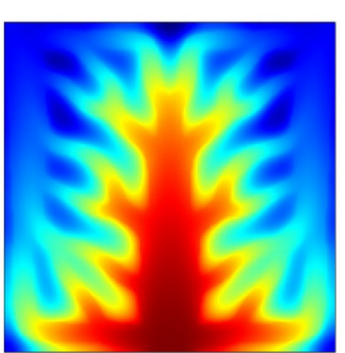

(c)

Oxygen mass fraction [-]
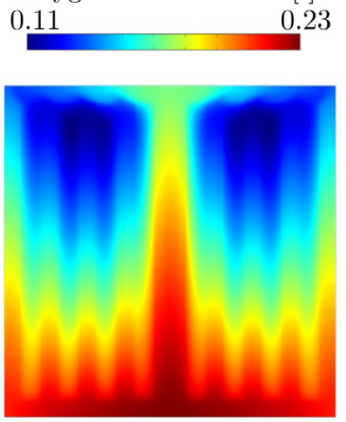

(f)

Oxygen mass fraction [-
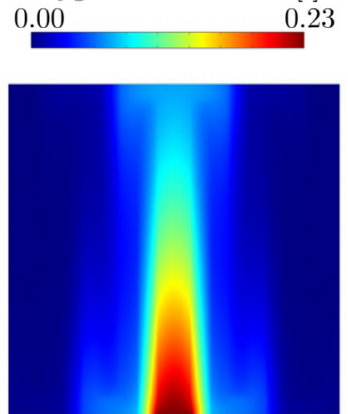

(i)

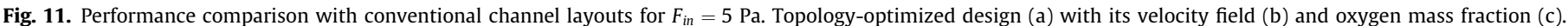
Parallel layout (d) with its velocity field (e) and oxygen mass fraction (f). Serpentine layout (g) with its velocity field (h) and oxygen mass fraction (i).

Table 6

Average current density $\left[\mathrm{A} / \mathrm{m}^{2}\right]$ for the three layouts at a comparable inlet pressure.

\begin{tabular}{ccc}
\hline Topology-optimized & Parallel & Serpentine \\
\hline 2785 & 2635 & 1120 \\
\hline
\end{tabular}

likely crucial for the scale-up of this lab-scale facility to industrial installations.

Finally, we note that the results obtained in the presented numerical example show that topology optimization allows the optimized flow paths in PEMFC to be obtained without the need for a close-to-optimal design to start with. The layout computed for a reference pressure drop of $F_{i n}=5 \mathrm{~Pa}$ is superior in terms of power generation to conventional alternatives like the serpentine and parallel configurations.

\subsection{The trade-off between pressure drop and power generation}

In this section, we analyze how the optimized designs and output power are affected by the choice of the inlet pressure, $F_{i n}$. The model and optimization parameters and physical properties are unchanged with respect to the reference design.

We consider 14 different values of the inlet pressure in the range $0.1 \mathrm{~Pa} \leqslant F_{\text {in }} \leqslant 40 \mathrm{~Pa}$. Only minor design and performance differences are observed for $F_{\text {in }}>40 \mathrm{~Pa}$. For each case, a topology optimization run is performed. To benchmark the effectiveness of our topology optimization framework, the parallel, serpentine and reference topology-optimized geometries considered in the previous section are analyzed in the same conditions. The computed average current density is shown in Fig. 13. In the range of values considered, a reduction in pressure drop can be obtained only at the expense of less power generation. Hence, these two per- 


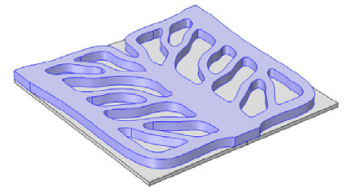

(a)

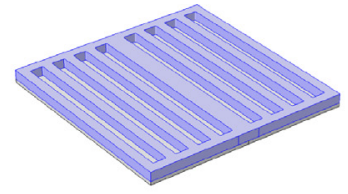

(b)

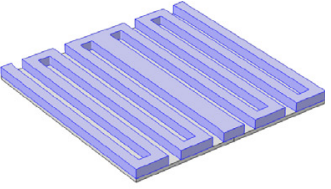

(c)

Fig. 12. 3D versions of the topology-optimized (a), parallel (b) and serpentine (c) designs.

Table 7

Performance of the 3D versions of the three layouts.

\begin{tabular}{lccc}
\hline \multirow{2}{*}{ Performance } & \multicolumn{3}{c}{ Design } \\
\cline { 2 - 4 } & $\begin{array}{c}\text { Topology- } \\
\text { optimized }\end{array}$ & Parallel & Serpentine \\
\hline Average current density $\left[\mathrm{A} / \mathrm{m}^{2}\right]$ & 2770 & 2661 & 1034 \\
2D deviation [\%] & -0.54 & +0.97 & -7.68 \\
\hline
\end{tabular}

Table 8

Comparison of blower consumption, pressure drop, and volumetric flow rate at a comparable current density.

\begin{tabular}{lccc}
\hline Performance & $\begin{array}{c}\text { Topology- } \\
\text { optimized }\end{array}$ & Parallel & Serpentine \\
\hline Average current density $\left[\mathrm{A} / \mathrm{m}^{2}\right]$ & 2785 & 2782 & 2777 \\
Pressure drop [Pa] & 5 & 10 & 40 \\
Volumetric flow rate $\left[\mathrm{m}^{3} / \mathrm{s}\right]\left(\times 10^{6}\right)$ & 1.61 & 2.40 & 1.65 \\
Blower consumption $[\mathrm{W}]\left(\times 10^{5}\right)$ & 1.01 & 3.00 & 8.26 \\
\hline
\end{tabular}

formance criteria are contradictory and the curve of the optimized performance in Fig. 13 is an approximation of the multi-objective Pareto front. The layouts obtained with our optimization framework are always superior to the conventional alternatives. Improvements are large at low inlet pressures. The average current density of the topology-optimized layout at $F_{\text {in }}=1 \mathrm{~Pa}$ is $83.21 \%$ and $574.8 \%$ higher than the one obtained using the parallel and the serpentine flow fields, respectively. This gap reduces for higher $F_{i n}$ so that the performance enhancement of the topologyoptimized layout is only $0.89 \%$ and $4.55 \%$ at $F_{i n}=40 \mathrm{~Pa}$. This suggests that the topology of the flow distributor slightly affects the performance at high inlet pressures. The reference topologyoptimized design yields always higher performance than the serpentine and parallel geometries and approximates well the Pareto front. Noticeable discrepancies are visible only at small pressure drops: for $F_{\text {in }}=1 \mathrm{~Pa}$, the reference topology-optimized design yields $13 \%$ lower performance than the layout optimized for that condition. However, also in this case negligible differences are observed at high inlet pressures.

The optimized layouts obtained at selected Pareto points are displayed in Fig. 14. When a higher inlet pressure is available, the geometry evolves more freely resulting in unintuitive layouts. The number of connections increases and the width of the channels decreases creating more and more complicated geometries. The optimized design for $F_{\text {in }}=40$ Pa shows similarities with the mesh design concept (Fig. 2)(d). To quantify the design modifications along the Pareto front, we study the evolution of two performance metrics. The first one is the number of internal holes, $N_{h}$, in the layout (filled by "porous rib" material) representing the complexity of the flow channel topology. The values obtained for the different inlet pressures are presented in the bar chart of Fig. 15(a). The number of internal holes, $N_{h}$, increases fast at low inlet pressures and levels-off at $F_{\text {in }}=20 \mathrm{~Pa}$. From this point onward, no topology modifications are observed. The second performance metric is a perimeter measure, $\Gamma_{p}$, based on the total variation of the material indicator field [69]:

$\Gamma_{p}=\int_{\Omega}\|\nabla \bar{s}\|_{2} d \mathbf{x}$

where $\|\cdot\|_{2}$ indicates the $\mathrm{L}_{2}$ norm. The influence of the inlet pressure on the perimeter measure of the optimized designs is shown in Fig. 15(b). The perimeter length increases monotonically in the range of values considered. At low inlet pressures, i.e. $F_{\text {in }} \leqslant 20 \mathrm{~Pa}$

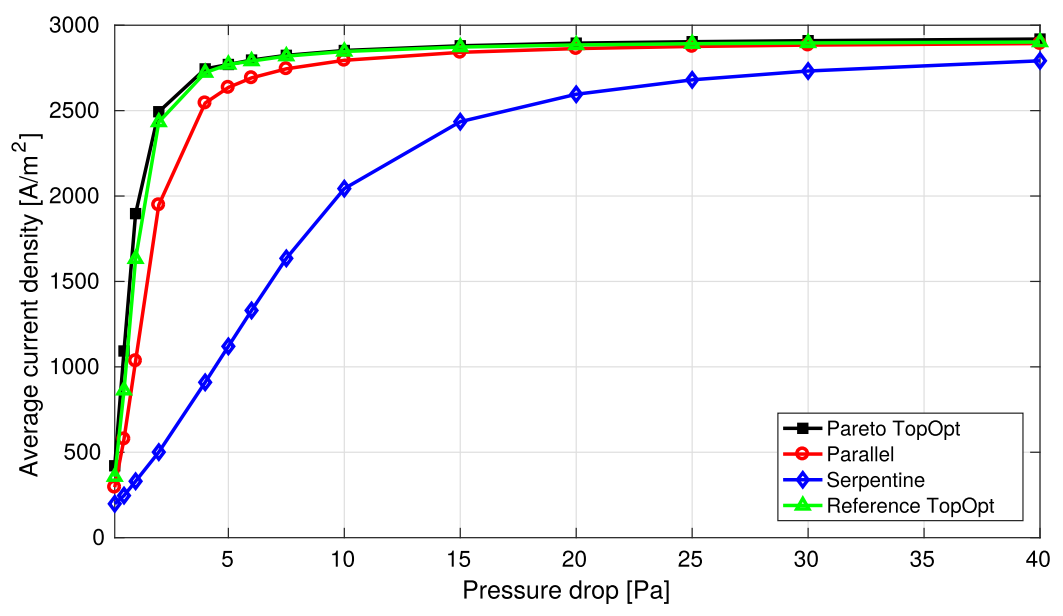

Fig. 13. Pareto front of the computed average current density versus pressure drop. 


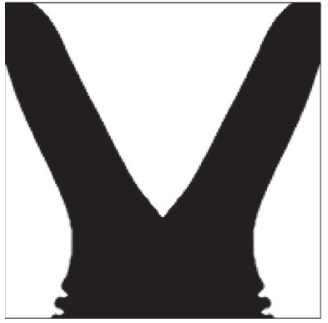

$0.1 \mathrm{~Pa}$

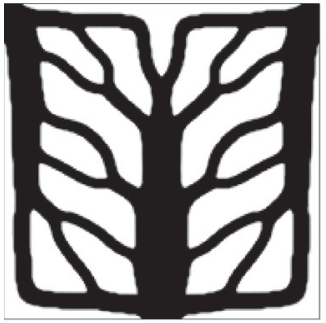

$5.0 \mathrm{~Pa}$

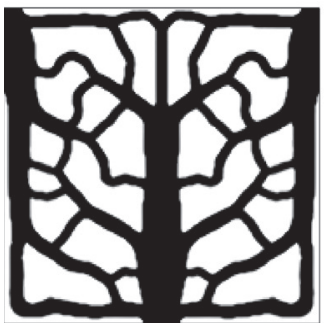

$15 \mathrm{~Pa}$

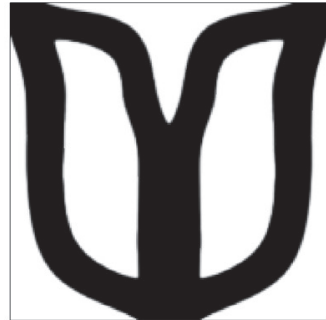

$1.0 \mathrm{~Pa}$

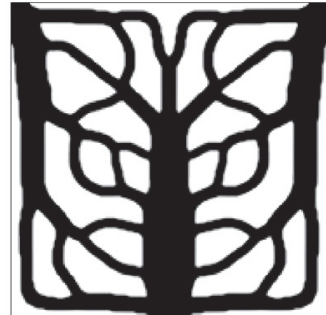

$7.5 \mathrm{~Pa}$

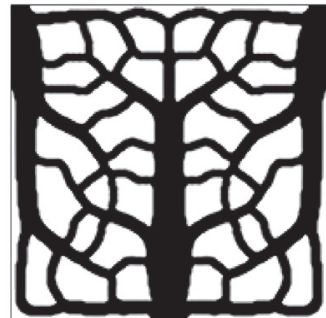

$20 \mathrm{~Pa}$

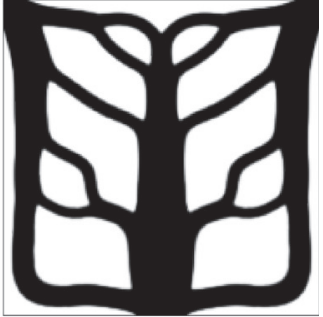

$2.0 \mathrm{~Pa}$

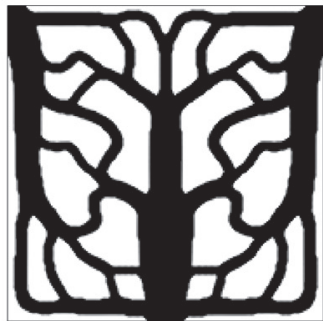

$10 \mathrm{~Pa}$

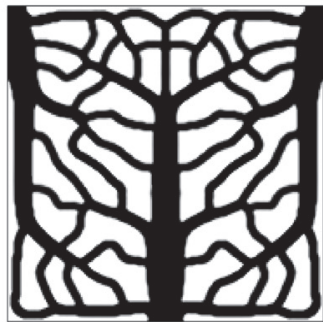

$40 \mathrm{~Pa}$

Fig. 14. Optimized designs obtained for different values of the imposed inlet pressure, $F_{\text {in }}$.

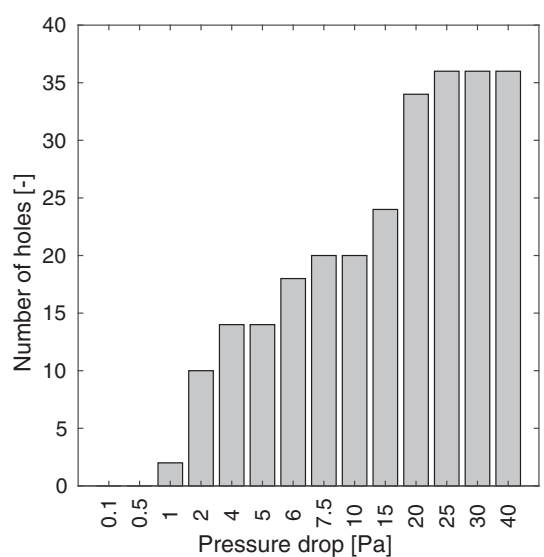

(a)

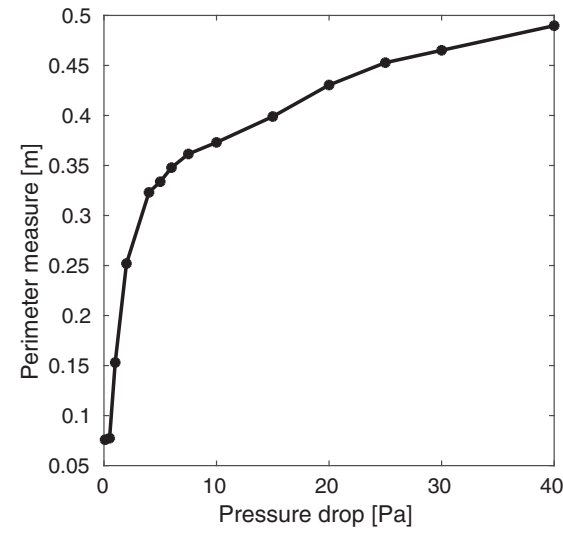

(b)

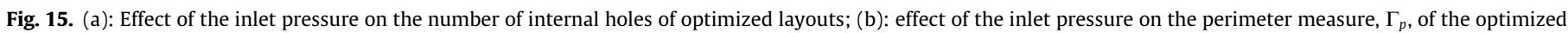
layouts.

corresponding to the region of topological changes, a fast growth of the perimeter measure is observed. For higher inlet pressure values, i.e. $F_{i n}>20 \mathrm{~Pa}, \Gamma_{p}$ is increased moderately as the perimeter modifications are obtained exclusively through a higher arc-to-chord ratio of the connections, resulting in a higher tortuosity of the channels.
This design study shows that the inlet pressure modifies consistently both the optimized designs and performance. At low inlet pressures, the topology complexity and power generation of the optimized designs increase fast. In this case, topology optimization yields layouts responsible for large improvements as compared to 


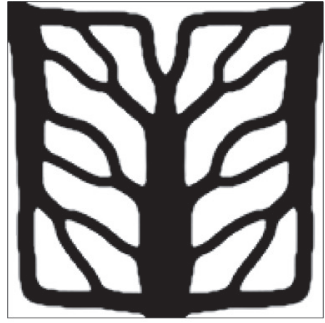

$w_{\theta}=0.00$

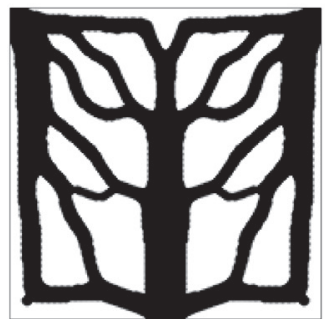

$w_{\theta}=2.00$

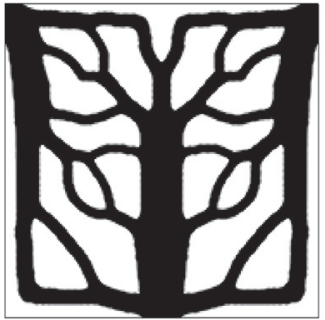

$w_{\theta}=0.25$

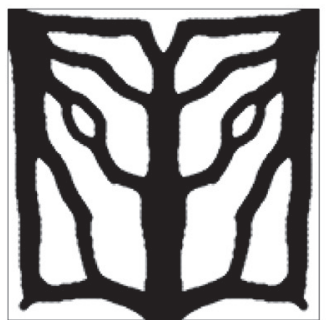

$w_{\theta}=5.00$

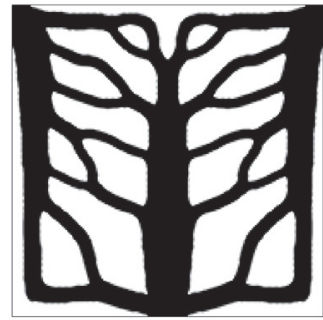

$w_{\theta}=0.50$

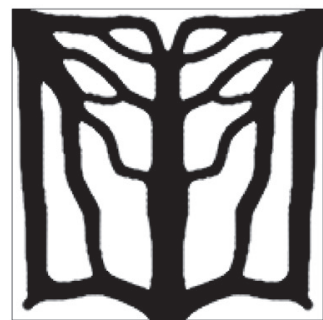

$w_{\theta}=7.50$

Fig. 16. Optimized designs obtained for different values of the current standard deviation weight, $w_{\theta}$, with $F_{\text {in }}=5$ Pa and $w_{\bar{W}}=1.00$.

conventional flow fields. At high inlet pressures, the performance is slightly affected by the layout of the gas distributors. The geometry changes are only due to tortuosity modifications of the channels, yielding minor power generation enhancements.

\subsection{Increasing the homogeneity of the current density distribution}

In this section, we study the design trends obtained using the full penalty method, i.e. $w_{\theta} \neq 0.00$ and $w_{\bar{w}} \neq 0.00$. The optimization problem (33) is solved for five values of the current standard deviation weight in the range $0.25 \leqslant w_{\theta} \leqslant 7.50$. Minor design and performance differences are observed for $w_{\theta}>7.50$. We consider the reference inlet pressure case, i.e. $F_{i n}=5 \mathrm{~Pa}$ and a fixed value of the power generation weight $w_{\bar{W}}=1.00$. The model parameters and material properties are unchanged with respect to the reference design. The optimized designs are shown in Fig. 16 along with the reference design case obtained by setting $w_{\theta}=0.00$. The results show that there is a significant influence of the weighting factor $w_{\theta}$ on the optimized layouts. As $w_{\theta}$ is increased, the geometries feature a higher concentration of "porous rib" material close to the inlets while the "porous channels" migrate towards the external edges and outlets. The vertical channel at the center of the cell gets thinner while two diagonal long and wide channels are created for $w_{\theta} \geqslant 5.00$, linking the inlet to the outlets. This suggests that more reactants are able to reach the regions close to the outlets through convective transport. To demonstrate these design trends, we consider the following performance metric:

$\widetilde{V}=\frac{\int_{\Omega_{2}} \bar{s} d \mathbf{x}}{\int_{\Omega_{1}} \bar{s} d \mathbf{x}}$,

where $\Omega_{1}$ and $\Omega_{2}$ are the portions of the design domain shown in Fig. 17(a). The parameter $\widetilde{V}$ quantifies the "density" of channels in the regions close to the outlets as compared to the region connected to the inlet. We will refer to $\widetilde{V}$ as volume ratio hereafter. The influ-

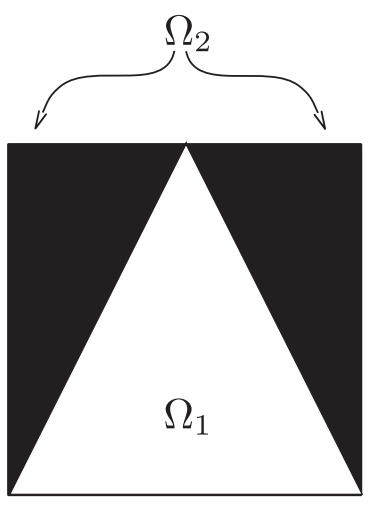

(a)

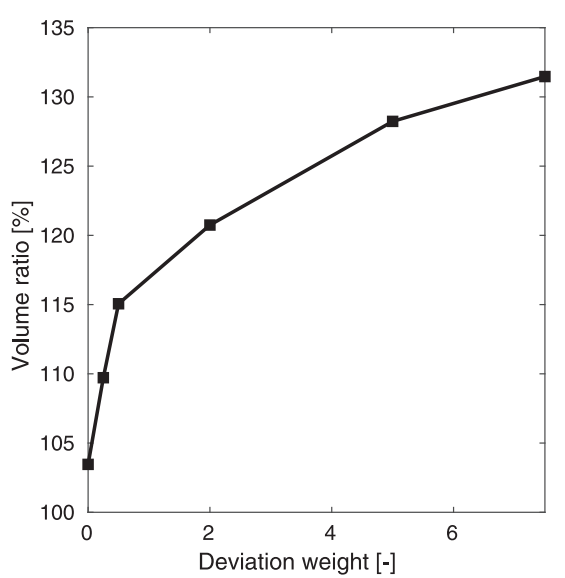

(b)

Fig. 17. (a): Domain splitting in close-to-inlet region $\left(\Omega_{1}\right)$ and close-to-outlets region $\left(\Omega_{2}\right)$; (b): effect of $w_{\theta}$ on volume ratio $\tilde{V}$. 


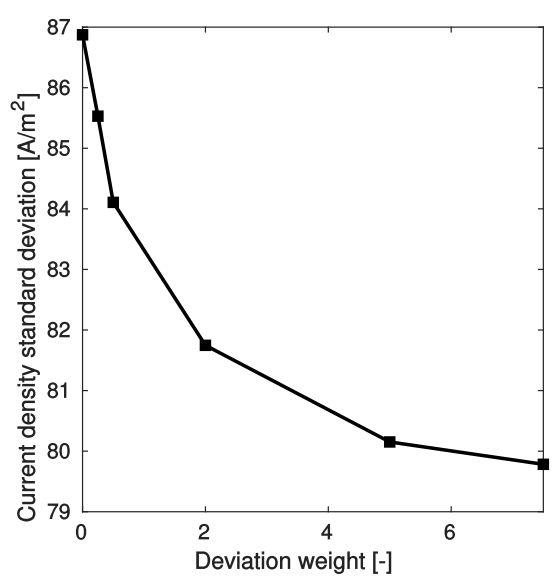

(a)

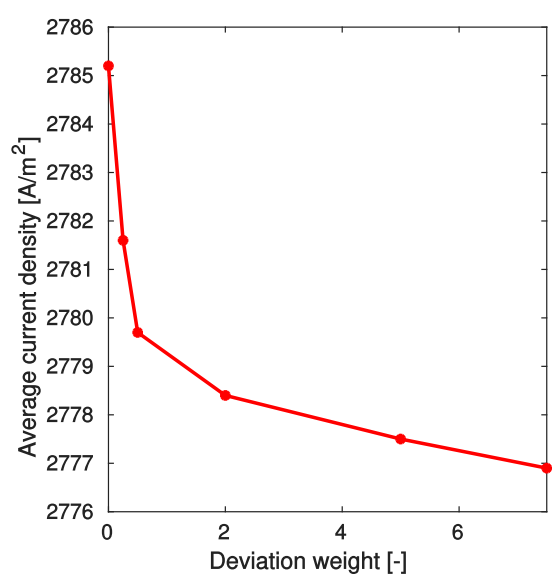

(b)

Fig. 18. (a): Effect of $w_{\bar{W}}$ on the standard deviation of current density; (b): effect of $w_{\bar{W}}$ on the average current density.

ence of $w_{\theta}$ on the volume ratio of the optimized layouts is shown in Fig. 17(b). In the range of values considered, $\tilde{V}$ increases monotonically. When the power is the unique optimization criteria $\left(w_{\theta}=0.00\right)$, the flow field complexity in $\Omega_{2}$ is comparable to that of $\Omega_{1}$ such that $\widetilde{V}=103.5 \%$. Obtaining a more homogeneous current density distribution requires more channels to be located close to the outlets. Setting $w_{\theta}=7.50$ yields a layout in which the total volume of the channels in $\Omega_{2}$ is $31.5 \%$ higher than the one measured in $\Omega_{1}$. Fig. 18(a) shows that these design modifications are effective in lowering our criterion of current density dispersion: $\theta_{i_{\text {loc }}}$ is decreased from $86.87 \mathrm{~A} / \mathrm{m}^{2}$ to $79.78 \mathrm{~A} / \mathrm{m}^{2}$, corresponding to a $8.16 \%$ reduction. The amount of power generated by the optimized

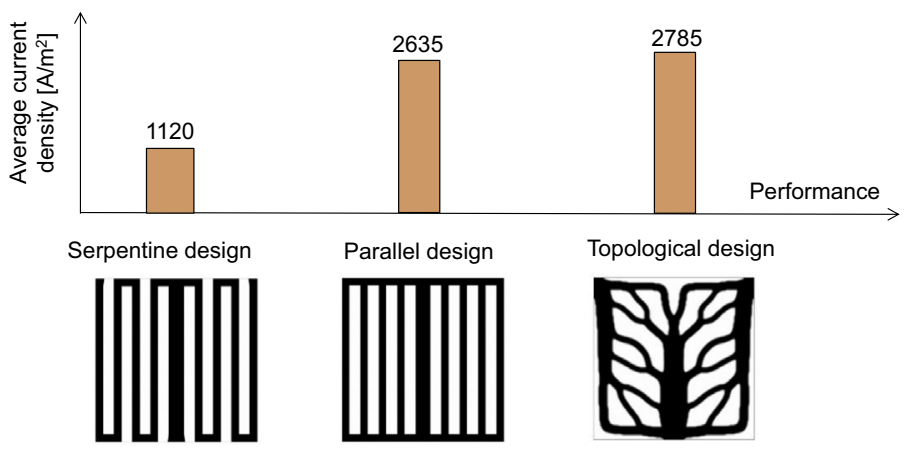

(a)

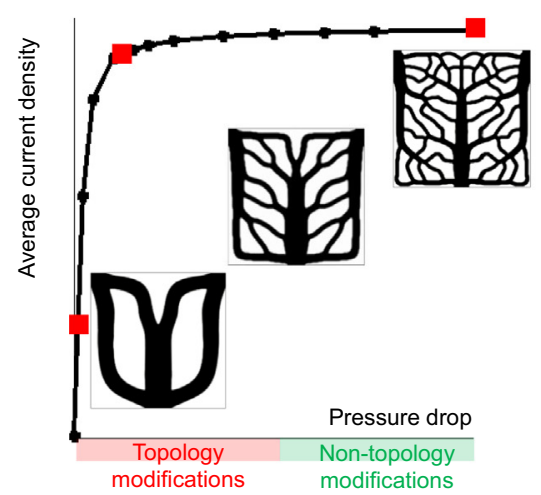

(b)

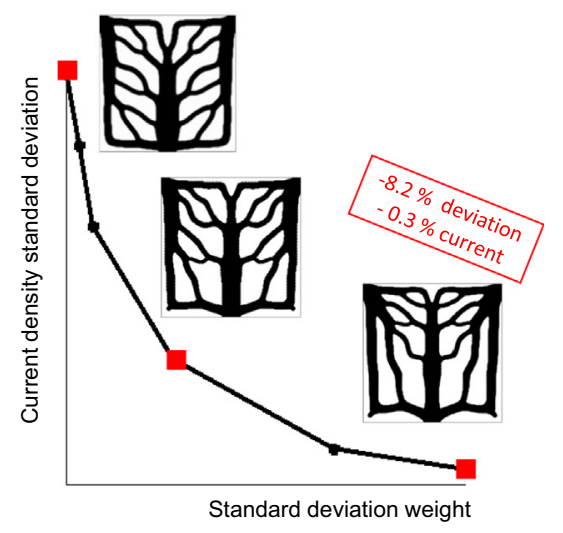

(c)

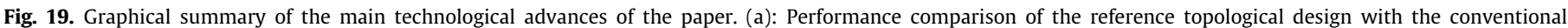

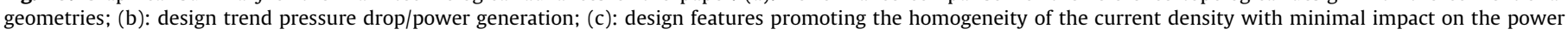
generation. 
layouts reveals to be slightly sensitive to the choice of $w_{\theta}$ (Fig. 18 (b)). We obtain a reduction of only $0.28 \%$ when raising $w_{\theta}$ from 0.00 to 7.50 , suggesting that the power generation is marginally affected by the design features promoting homogeneity. This indicates that the inclusion of current density dispersion measures in flow field design optimization frameworks has the potential to indicate promising routes for practical technological development.

\section{Conclusions}

This paper demonstrated the use of density-based topology optimization for the practical design of flow fields for PEMFCs. The objective function was formulated to maximize both the output power and homogeneity of the current density distribution, permitting reduced costs and higher durability. The gas dynamics, transport of chemical species, and electrochemical interactions in the cathodic portion of the PEMFC were computed using a 2D finite element model. The 3D transport effects were accounted for in the 2D model through an original depth-averaging procedure, which was verified against the predictions of a full 3D model calibrated using experimental and numerical results available in literature.

A graphical summary of the main technological advances is presented in Fig. 19. Topology optimization yielded layouts with unintuitive and non-trivial features. Our reference flow field obtained considering an inlet pressure of $5 \mathrm{~Pa}$ has similarities with various design concepts such as parallel, mesh, and bio-inspired geometries. Its performance was found to be superior to one of both parallel and serpentine flow fields, yielding a $5.7 \%$ and a $248.6 \%$ increase in the average current density (Fig. 19(a)). The smaller fluid-dynamic resistance of our layout allows a much higher air flow rate as compared to the serpentine geometry. Furthermore, its effective reactants distribution avoids the creation of regions with a large oxygen depletion, which arises in the parallel geometry. The trade-off between pressure drop and power generation was then explored to study the influence of the inlet pressure on both the optimized designs and performance (Fig. 19(b)). The results showed that, at low inlet pressures, substantial power output improvements are achievable through topological modifications of the flow distributor. In this case, the optimization process yielded large enhancements with respect to the conventional flow fields. At higher inlet pressures, no topology modifications were observed and only moderate enhancements could be obtained through the increase of the tortuosity of the channels. In this case, the performance was found to be slightly sensitive to the flow field layout and the topology-optimized designs performed similarly to the serpentine and parallel flow fields. Adding a measure of the current density dispersion to the optimization objective led to design features capable of decreasing the current density standard deviation by $8.16 \%$ with negligible impact on the amount of power generated (Fig. 19(c)). This finding suggests a viable route for the future development of these systems with the aim of increasing the durability.

The developed framework can largely impact the technological development of electrochemical systems. Future studies will focus on the 3D multi-scale topology optimization of gas diffusion media with optimized size and shape of the pores. The combination of this design capability with a multi-phase analysis model will allow the realization of devices with patterned wettability [86-88] with advanced water management. Furthermore, including multi-phase flow in the current analysis and design framework has the potential to yield flow field features that maximize water removal through under-rib transport. Finally, the extension of this framework to the optimization of flow fields in redox flow batteries [89-91] can boost the unexplored potential of this emerging technology and deserves attention in future research.

\section{Conflict of interest}

We confirm that we have no conflicts of interest to disclose.

\section{Acknowledgments}

The authors gratefully acknowledge generous help of the University of California San Diego Digital Media Lab (DML) in 3Dprinting of the design sample. AFC gratefully acknowledges the financial support of the Swiss National Science Foundation (P2EZP2_172183).

\section{References}

[1] L. Carrette, K.A. Friedrich, U. Stimming, Fuel cells - fundamentals and applications, Fuel Cells 1 (1) (2001) 5-39, https://doi.org/10.1002/1615-6854 (200105) $1: 1<5:$ :aid-fuce $5>3.0 . c 0 ; 2-\mathrm{g}$.

[2] J.-H. Wee, Applications of proton exchange membrane fuel cell systems, Renew. Sustain. Energy Rev. 11 (8) (2007) 1720-1738, https://doi.org/10.1016/ j.rser.2006.01.005.

[3] S.-J. Cheng, J.-M. Miao, S.-J. Wu, Use of metamodeling optimal approach promotes the performance of proton exchange membrane fuel cell (PEMFC), Appl. Energy 105 (2013) 161-169, https://doi.org/10.1016/j. apenergy.2013.01.001.

[4] A. Aiyejina, M.K.S. Sastry, PEMFC flow channel geometry optimization: a review, J. Fuel Cell Sci. Technol. 9 (1) (2012) 011011, https://doi.org/10.1115/ 1.4005393.

[5] A. Kumar, R.G. Reddy, Effect of channel dimensions and shape in the flow-field distributor on the performance of polymer electrolyte membrane fuel cells, J. Power Sources 113 (1) (2003) 11-18, https://doi.org/10.1016/s0378-7753(02) 00475-5.

[6] Y. Wang, K.S. Chen, J. Mishler, S.C. Cho, X.C. Adroher, A review of polymer electrolyte membrane fuel cells: technology, applications, and needs on fundamental research, Appl. Energy 88 (4) (2011) 981-1007, https://doi.org/ 10.1016/j.apenergy.2010.09.030.

[7] A. Arvay, J. French, J.-C. Wang, X.-H. Peng, A. Kannan, Nature inspired flow field designs for proton exchange membrane fuel cell, Int. J. Hydrogen Energy 38 (9) (2013) 3717-3726, https://doi.org/10.1016/j.ijhydene.2012.12.149.

[8] B. Lim, E. Majlan, W. Daud, M. Rosli, T. Husaini, Numerical analysis of modified parallel flow field designs for fuel cells, Int. J. Hydrogen Energy 42 (14) (2017) 9210-9218, https://doi.org/10.1016/j.ijhydene.2016.03.189.

[9] A. Kopanidis, A. Theodorakakos, M. Gavaises, D. Bouris, Pore scale 3d modelling of heat and mass transfer in the gas diffusion layer and cathode channel of a PEM fuel cell, Int. J. Therm. Sci. 50 (4) (2011) 456-467, https://doi.org/10.1016/ j.ijthermalsci.2010.11.014.

[10] A.-R. Kim, S. Shin, S. Um, Multidisciplinary approaches to metallic bipolar plate design with bypass flow fields through deformable gas diffusion media of polymer electrolyte fuel cells, Energy 106 (2016) 378-389, https://doi.org/ 10.1016/j.energy.2016.03.073.

[11] C. Wang, Q. Zhang, S. Shen, X. Yan, F. Zhu, X. Cheng, J. Zhang, The respective effect of under-rib convection and pressure drop of flow fields on the performance of PEM fuel cells, Sci. Rep. 7 (2017) 43447, https://doi.org/ $10.1038 /$ srep43447.

[12] N. Zehtabiyan-Rezaie, A. Arefian, M.J. Kermani, A.K. Noughabi, M. Abdollahzadeh, Effect of flow field with converging and diverging channels on proton exchange membrane fuel cell performance, Energy Convers. Manage. 152 (2017) 31-44, https://doi.org/10.1016/j.enconman.2017.09.009.

[13] A. Manso, F. Marzo, J. Barranco, X. Garikano, M.G. Mujika, Influence of geometric parameters of the flow fields on the performance of a PEM fuel cell. A review, Int. J. Hydrogen Energy 37 (20) (2012) 15256-15287, https://doi.org/ 10.1016/j.ijhydene.2012.07.076.

[14] E. Afshari, M. Mosharaf-Dehkordi, H. Rajabian, An investigation of the PEM fuel cells performance with partially restricted cathode flow channels and metal foam as a flow distributor, Energy 118 (2017) 705-715, https://doi.org/ 10.1016/j.energy.2016.10.101.

[15] Y. FERNG, A. SU, A three-dimensional full-cell CFD model used to investigate the effects of different flow channel designs on PEMFC performance, Int. J. Hydrogen Energy 32 (17) (2007) 4466-4476, https://doi.org/10.1016/j. ijhydene.2007.05.012.

[16] J. Owejan, T. Trabold, D. Jacobson, M. Arif, S. Kandlikar, Effects of flow field and diffusion layer properties on water accumulation in a PEM fuel cell, Int. J. Hydrogen Energy 32 (17) (2007) 4489-4502, https://doi.org/10.1016/j. ijhydene.2007.05.044.

[17] L.S. Freire, E. Antolini, M. Linardi, E.I. Santiago, R.R. Passos, Influence of operational parameters on the performance of PEMFCs with serpentine flow field channels having different (rectangular and trapezoidal) cross-section shape, Int. J. Hydrogen Energy 39 (23) (2014) 12052-12060, https://doi.org/ 10.1016/j.ijhydene.2014.06.041.

[18] X. Zeng, Y. Ge, J. Shen, L. Zeng, Z. Liu, W. Liu, The optimization of channels for a proton exchange membrane fuel cell applying genetic algorithm, Int. J. Heat Mass Transf. $105 \quad$ (2017) 81-89, https://doi.org/10.1016/j. ijheatmasstransfer.2016.09.068. 
[19] W.-M. Yan, H.-C. Liu, C.-Y. Soong, F. Chen, C. Cheng, Numerical study on cell performance and local transport phenomena of PEM fuel cells with novel flow field designs, J. Power Sources 161 (2) (2006) 907-919, https://doi.org/ 10.1016/j.jpowsour.2006.05.007.

[20] H. Liu, W. Yan, C. Soong, F. Chen, H. Chu, Reactant gas transport and cell performance of proton exchange membrane fuel cells with tapered flow field design, J. Power Sources 158 (1) (2006) 78-87, https://doi.org/10.1016/j. jpowsour.2005.09.017.

[21] C.-H. Huang, J.-W. Lin, Optimal gas channel shape design for a serpentine PEMFC: theoretical and experimental studies, J. Electrochem. Soc. 156 (1) (2009) B178, https://doi.org/10.1149/1.3025296.

[22] C.-H. Huang, L.-Y. Chen, S. Kim, An inverse geometry design problem in optimizing the shape of the gas channel for a proton exchange membrane fuel cell, J. Power Sources 187 (1) (2009) 136-147, https://doi.org/10.1016/j. jpowsour.2008.10.093.

[23] X.-D. Wang, Y.-X. Huang, C.-H. Cheng, J.-Y. Jang, D.-J. Lee, W.-M. Yan, A. Su, An inverse geometry design problem for optimization of single serpentine flow field of PEM fuel cell, Int. J. Hydrogen Energy 35 (9) (2010) 4247-4257, https:// doi.org/10.1016/j.ijhydene.2010.02.059.

[24] E. Mancusi, É. Fontana, A.A.U. de Souza, S.M.G.U. de Souza, Numerical study of two-phase flow patterns in the gas channel of PEM fuel cells with tapered flow field design, Int. J. Hydrogen Energy 39 (5) (2014) 2261-2273, https://doi.org/ 10.1016/j.ijhydene.2013.11.106.

[25] M. Secanell, J. Wishart, P. Dobson, Computational design and optimization of fuel cells and fuel cell systems: a review, J. Power Sources 196 (8) (2011) 3690-3704, https://doi.org/10.1016/j.jpowsour.2010.12.011.

[26] B.H. Lim, E.H. Majlan, W.R.W. Daud, T. Husaini, M.I. Rosli, Effects of flow field design on water management and reactant distribution in PEMFC: a review, Ionics 22 (3) (2016) 301-316, https://doi.org/10.1007/s11581-016-1644-y.

[27] T. Monsaf, B.M. Hocine, S. Youcef, M. Abdallah, Unsteady three-dimensional numerical study of mass transfer in PEM fuel cell with spiral flow field, Int. J. Hydrogen Energy 42 (2) (2017) 1237-1251, https://doi.org/10.1016/j. ijhydene.2016.12.084.

[28] E. Alizadeh, M. Rahimi-Esbo, S. Rahgoshay, S. Saadat, M. Khorshidian, Numerical and experimental investigation of cascade type serpentine flow field of reactant gases for improving performance of PEM fuel cell, Int. J. Hydrogen Energy 42 (21) (2017) 14708-14724, https://doi.org/10.1016/j. ijhydene.2017.04.212.

[29] P. Karthikeyan, R. Vasanth, M. Muthukumar, Experimental investigation on uniform and zigzag positioned porous inserts on the rib surface of cathode flow channel for performance enhancement in PEMFC, Int. J. Hydrogen Energy 40 (13) (2015) 4641-4648, https://doi.org/10.1016/j.ijhydene.2015.01.175.

[30] M. Seyhan, Y.E. Akansu, M. Murat, Y. Korkmaz, S.O. Akansu, Performance prediction of PEM fuel cell with wavy serpentine flow channel by using artificial neural network, Int. J. Hydrogen Energy 42 (40) (2017) 25619-25629, https://doi.org/10.1016/j.ijhydene.2017.04.001.

[31] M.Z. Chowdhury, Y.E. Akansu, Novel convergent-divergent serpentine flow fields effect on PEM fuel cell performance, Int. J. Hydrogen Energy 42 (40) (2017) 25686-25694, https://doi.org/10.1016/j.ijhydene.2017.04.079.

[32] G.M. Imbrioscia, H.J. Fasoli, Simulation and study of proposed modifications over straight-parallel flow field design, Int. J. Hydrogen Energy 39 (16) (2014) 8861-8867, https://doi.org/10.1016/j.ijhydene.2013.11.079.

[33] B.-T. Tsai, C.-J. Tseng, Z.-S. Liu, C.-H. Wang, C.-I. Lee, C.-C. Yang, S.-K. Lo, Effects of flow field design on the performance of a PEM fuel cell with metal foam as the flow distributor, Int. J. Hydrogen Energy 37 (17) (2012) 13060-13066, https://doi.org/10.1016/j.ijhydene.2012.05.008.

[34] S. Huo, N.J. Cooper, T.L. Smith, J.W. Park, K. Jiao, Experimental investigation on PEM fuel cell cold start behavior containing porous metal foam as cathode flow distributor, Appl. Energy 203 (2017) 101-114, https://doi.org/10.1016/j. apenergy.2017.06.028

[35] N. Konno, S. Mizuno, H. Nakaji, Y. Ishikawa, Development of compact and highperformance fuel cell stack, SAE Int. J. Altern. Powertrains 4 (1)(2015), https:// doi.org/10.4271/2015-01-1175.

[36] J.P. Kloess, X. Wang, J. Liu, Z. Shi, L. Guessous, Investigation of bio-inspired flow channel designs for bipolar plates in proton exchange membrane fuel cells, J. Power Sources 188 (1) (2009) 132-140, https://doi.org/10.1016/j. jpowsour.2008.11.123.

[37] R. Roshandel, F. Arbabi, G.K. Moghaddam, Simulation of an innovative flowfield design based on a bio inspired pattern for PEM fuel cells, Renew. Energy 41 (2012) 86-95, https://doi.org/10.1016/j.renene.2011.10.008.

[38] C.E. Damian-Ascencio, A. Saldaña-Robles, A. Hernandez-Guerrero, S. CanoAndrade, Numerical modeling of a proton exchange membrane fuel cell with tree-like flow field channels based on an entropy generation analysis, Energy 133 (2017) 306-316, https://doi.org/10.1016/j.energy.2017.05.139.

[39] X. Guo, W. Zhang, W. Zhong, Doing topology optimization explicitly and geometrically-a new moving morphable components based framework, J Appl. Mech. 81 (8) (2014) 081009, https://doi.org/10.1115/1.4027609.

[40] A. Pizzolato, A. Sharma, K. Maute, A. Sciacovelli, V. Verda, Topology optimization for heat transfer enhancement in latent heat thermal energy storage, Int. J. Heat Mass Transf. 113 (2017) 875-888, https://doi.org/10.1016/ j.ijheatmasstransfer.2017.05.098.

[41] A. Pizzolato, A. Sharma, K. Maute, A. Sciacovelli, V. Verda, Design of effective fins for fast PCM melting and solidification in shell-and-tube latent heat thermal energy storage through topology optimization, Appl. Energy (2017), https://doi.org/10.1016/j.apenergy.2017.10.050.
[42] R. Behrou, M. Lawry, K. Maute, Level set topology optimization of structural problems with interface cohesion, Int. J. Numer. Meth. Eng. 112 (8) (2017) 990-1016, https://doi.org/10.1002/nme.5540.

[43] R. Behrou, J.K. Guest, Topology optimization for transient response of structures subjected to dynamic loads, in: 18th AIAA/ISSMO Multidisciplinary Analysis and Optimization Conference, 2017, https://doi. org/10.2514/6.2017-3657.

[44] C. Kim, H. Sun, Topology optimization of gas flow channel routes in an automotive fuel cell, Int. J. Automotive Technol. 13 (5) (2012) 783-789, https://doi.org/10.1007/s12239-012-0078-4.

[45] G. Zhang, L. Guo, B. Ma, H. Liu, Comparison of current distributions in proton exchange membrane fuel cells with interdigitated and serpentine flow fields, J. Power Sources 188 (1) (2009) 213-219.

[46] C. Siegel, Review of computational heat and mass transfer modeling in polymer-electrolyte-membrane (pem) fuel cells, Energy 33 (9) (2008) 13311352

[47] F.C. Cetinbas, S.G. Advani, A.K. Prasad, Three dimensional proton exchange membrane fuel cell cathode model using a modified agglomerate approach based on discrete catalyst particles, J. Power Sources 250 (2014) 110-119, https://doi.org/10.1016/j.jpowsour.2013.10.138.

[48] Z. He, H. Li, K.E. Birgersson, Reduced Modelling of Planar Fuel Cells, Springer International Publishing, 2017.

[49] Y. Zhou, G. Lin, A. Shih, S. Hu, A micro-scale model for predicting contact resistance between bipolar plate and gas diffusion layer in pem fuel cells, J. Power Sources 163 (2) (2007) 777-783, https://doi.org/10.1016/j. jpowsour.2006.09.019.

[50] X. Lai, L. Peng, J. Ni, et al., A mechanical-electrical finite element method model for predicting contact resistance between bipolar plate and gas diffusion layer in pem fuel cells, J. Power Sources 182 (1) (2008) 153-159, https://doi.org/10.1016/j.jpowsour.2008.03.069.

[51] P. Liang, D. Qiu, L. Peng, P. Yi, X. Lai, J. Ni, Contact resistance prediction of proton exchange membrane fuel cell considering fabrication characteristics of metallic bipolar plates, Energy Convers. Manage. 169 (2018) 334-344, https:// doi.org/10.1016/j.enconman.2018.05.069.

[52] E. Sanchez-Palencia, A. Zaoui (Eds.), Homogenization Techniques for Composite Media, Springer, Berlin, Heidelberg, 1987, https://doi.org/10.1007/ 3-540-17616-0.

[53] P. Renard, G. de Marsily, Calculating equivalent permeability: a review, Adv. Water Resour. 20 (5-6) (1997) 253-278, https://doi.org/10.1016/s0309-1708 (96)00050-4.

[54] G. Allaire, A brief introduction to homogenization and miscellaneous applications, ESAIM: Proceedings 37 (2012) 1-49, https://doi.org/10.1051/ proc/201237001.

[55] L.H. Olesen, F. Okkels, H. Bruus, A high-level programming-language implementation of topology optimization applied to steady-state navierstokes flow, Int. J. Numer. Meth. Eng. 65 (7) (2006) 975-1001, https://doi.org/ 10.1002/nme.1468.

[56] T. Borrvall, J. Petersson, Topology optimization of fluids in stokes flow, Int. J. Numer. Meth. Fluids 41 (1) (2002) 77-107, https://doi.org/10.1002/fld.426.

[57] R.J. Kee, M.E. Coltrin, P. Glarborg, Chemically Reacting Flow, John Wiley \& Sons Inc., 2003, https://doi.org/10.1002/0471461296.

[58] R. Krishna, J. Wesselingh, The maxwell-stefan approach to mass transfer, Chem. Eng. Sci. 52 (6) (1997) 861-911, https://doi.org/10.1016/s0009-2509 (96)00458-7.

[59] V. Fontalvo, D. Illera, H. Gómez, M. Sanjuan, CFD multiphysics modeling and performance evaluation of PEM fuel cells, in: Volume 6: Energy, ASME, 2017, https://doi.org/10.1115/imece2017-72160.

[60] M. Lindstrom, B. Wetton, A comparison of fick and Maxwell-Stefan diffusion formulations in PEMFC gas diffusion layers, Heat Mass Transf. 53 (1) (2016) 205-212, https://doi.org/10.1007/s00231-016-1812-7.

[61] R. O'Hayre, S.-W. Cha, W. Colella, F.B. Prinz, Fuel Cell Fundamentals, John Wiley \& Sons Inc, 2016, https://doi.org/10.1002/9781119191766.

[62] J. Larminie, A. Dicks, Fuel cell systems analysed, in: Fuel Cell Systems Explained, John Wiley \& Sons Ltd., 2013, pp. 369-389, https://doi.org/ 10.1002/9781118878330.ch11.

[63] C. Multiphysics, v. 5.3, COMSOL AB, Stockholm, Sweden, 2017.

[64] T.J. Hughes, L.P. Franca, M. Balestra, A new finite element formulation for computational fluid dynamics: V. Circumventing the babuška-brezzi condition: a stable petrov-galerkin formulation of the stokes problem accommodating equal-order interpolations, Comput. Methods Appl. Mech. Eng. 59 (1) (1986) 85-99, https://doi.org/10.1016/0045-7825(86)90025-3.

[65] T. Tezduyar, S. Mittal, S. Ray, R. Shih, Incompressible flow computations with stabilized bilinear and linear equal-order-interpolation velocity-pressure elements, Comput. Methods Appl. Mech. Eng. 95 (2) (1992) 221-242, https://doi.org/10.1016/0045-7825(92)90141-6.

[66] G. Hauke, T. Hughes, A unified approach to compressible and incompressible flows, Comput. Methods Appl. Mech. Eng. 113 (3-4) (1994) 389-395, https:// doi.org/10.1016/0045-7825(94)90055-8.

[67] R. Codina, A discontinuity-capturing crosswind-dissipation for the finite element solution of the convection-diffusion equation, Comput. Methods Appl. Mech. Eng. 110 (3-4) (1993) 325-342, https://doi.org/10.1016/00457825(93)90213-h.

[68] P.R. Amestoy, I.S. Duff, J.-Y. L'Excellent, J. Koster, A fully asynchronous multifrontal solver using distributed dynamic scheduling, SIAM J. Matrix Anal. Appl. 23 (1) (2001) 15-41, https://doi.org/10.1137/s0895479899358194. 
[69] O. Sigmund, K. Maute, Topology optimization approaches, Struct. Multidiscipl. Optim. 48 (6) (2013) 1031-1055, https://doi.org/10.1007/s00158-013-0978-6.

[70] M.P. Bendsøe, O. Sigmund, Topology Optimization: Theory, Methods and Applications, Springer, Berlin, Heidelberg, 2004, https://doi.org/10.1007/9783-662-05086-6.

[71] J. Alexandersen, N. Aage, C.S. Andreasen, O. Sigmund, Topology optimisation for natural convection problems, Int. J. Numer. Meth. Fluids 76 (10) (2014) 699-721, https://doi.org/10.1002/fld.3954.

[72] J. Alexandersen, O. Sigmund, N. Aage, Large scale three-dimensional topology optimisation of heat sinks cooled by natural convection, Int. J. Heat Mass

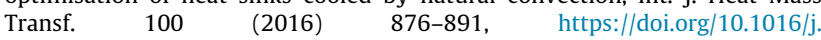
ijheatmasstransfer.2016.05.013.

[73] O. Sigmund, J. Petersson, Numerical instabilities in topology optimization: a survey on procedures dealing with checkerboards, mesh-dependencies and local minima, Struct. Optim. 16 (1) (1998) 68-75, https://doi.org/10.1007/ bf01214002.

[74] F. Wang, B.S. Lazarov, O. Sigmund, On projection methods, convergence and robust formulations in topology optimization, Struct. Multidiscipl. Optim. 43 (6) (2010) 767-784, https://doi.org/10.1007/s00158-010-0602-y.

[75] J.K. Guest, J.H. Prévost, T. Belytschko, Achieving minimum length scale in topology optimization using nodal design variables and projection functions, Int. J. Numer. Meth. Eng. 61 (2) (2004) 238-254, https://doi.org/10.1002/ nme.1064.

[76] K. Svanberg, A class of globally convergent optimization methods based on conservative convex separable approximations, SIAM J. Optim. 12 (2) (2002) 555-573, https://doi.org/10.1137/s1052623499362822.

[77] J.K. Guest, A. Asadpoure, S.-H. Ha, Eliminating beta-continuation from heaviside projection and density filter algorithms, Struct. Multidiscipl. Optim. 44 (4) (2011) 443-453, https://doi.org/10.1007/s00158-011-0676-1.

[78] M. Haghayegh, M.H. Eikani, S. Rowshanzamir, Modeling and simulation of a proton exchange membrane fuel cell using computational fluid dynamics, Int. J. Hydrogen Energy 42 (34) (2017) 21944-21954, https://doi.org/10.1016/j. ijhydene.2017.07.098.

[79] D. Nield, A. Bejan, Convection in porous media, in: Convection Heat Transfer, John Wiley \& Sons Inc., 2013, pp. 537-605, https://doi.org/10.1002/ 9781118671627.ch12.

[80] U.R. Salomov, E. Chiavazzo, P. Asinari, Gas-dynamic and electro-chemical optimization of catalyst layers in high temperature polymeric electrolyte membrane fuel cells, Int. J. Hydrogen Energy 40 (15) (2015) 5425-5431, https://doi.org/10.1016/j.ijhydene.2015.01.059.
[81] A. Chandan, M. Hattenberger, A. El-kharouf, S. Du, A. Dhir, V. Self, B.G. Pollet, A Ingram, W. Bujalski, High temperature (HT) polymer electrolyte membrane fuel cells (PEMFC) - a review, J. Power Sources 231 (2013) 264-278, https:// doi.org/10.1016/j.jpowsour.2012.11.126.

[82] H. Ruan, C. Wu, S. Liu, T. Chen, Design and simulation of novel flow field plate geometry for proton exchange membrane fuel cells, Heat Mass Transf. 52 (10) (2015) 2167-2176, https://doi.org/10.1007/s00231-015-1737-6.

[83] H. Kahraman, M.F. Orhan, Flow field bipolar plates in a proton exchange membrane fuel cell: analysis \& modeling, Energy Convers. Manage. 133 (2017) 363-384, https://doi.org/10.1016/j.enconman.2016.10.053.

[84] R. Taccani, N. Zuliani, Effect of flow field design on performances of high temperature PEM fuel cells: experimental analysis, Int. J. Hydrogen Energy 36 (16) (2011) 10282-10287, https://doi.org/10.1016/j.ijhydene.2010.10.026.

[85] J. Wang, Theory and practice of flow field designs for fuel cell scaling-up: a critical review, Appl. Energy 157 (2015) 640-663, https://doi.org/10.1016/j. apenergy.2015.01.032.

[86] A. Forner-Cuenca, J. Biesdorf, L. Gubler, P.M. Kristiansen, T.J. Schmidt, P. Boillat Engineered water highways in fuel cells: radiation grafting of gas diffusion layers, Adv. Mater. 27 (41) (2015) 6317-6322, https://doi.org/10.1002/ adma.201503557.

[87] A. Forner-Cuenca, J. Biesdorf, V. Manzi-Orezzoli, L. Gubler, T.J. Schmidt, P. Boillat, Advanced water management in pefcs: diffusion layers with patterned wettability iii. operando characterization with neutron imaging, J Electrochem. Soc. 163 (13) (2016) F1389-F1398, https://doi.org/10.1149/ 2.0891613jes.

[88] A. Forner-Cuenca, V. Manzi-Orezzoli, P.M. Kristiansen, L. Gubler, TJ Schmidt, P. Boillat, Mask-assisted electron radiation grafting for localized throughvolume modification of porous substrates: influence of electron energy on spatial resolution, Radiat. Phys. Chem. 135 (2017) 133-141, https://doi.org 10.1016/j.radphyschem.2017.01.036.

[89] A.Z. Weber, M.M. Mench, J.P. Meyers, P.N. Ross, J.T. Gostick, Q. Liu, Redox flow batteries: a review, J. Appl. Electrochem. 41 (10) (2011) 1137, https://doi.org 10.1007/s10800-011-0348-2.

[90] R.M. Darling, M.L. Perry, The influence of electrode and channel configurations on flow battery performance, J. Electrochem. Soc. 161 (9) (2014) A1381A1387, https://doi.org/10.1149/2.0941409jes.

[91] C.-H. Chen, K. Yaji, S. Yamasaki, S. Tsushima, K. Fujita, Novel flow field design for vanadium redox flow batteries via topology optimization, arXiv preprint arXiv:1807.07764doi:arXiv:1807.07764. 